\title{
Perturbative studies of toroidal momentum transport using neutral beam injection modulation in the Joint European Torus: Experimental results, analysis methodology, and first principles modeling
}

\author{
P. Mantica, ${ }^{1}$ T. Tala, ${ }^{2}$ J. S. Ferreira, ${ }^{3}$ A. G. Peeters, ${ }^{4}$ A. Salmi, ${ }^{5}$ D. Strintzi, ${ }^{6}$ J. Weiland, ${ }^{7}$ \\ M. Brix, ${ }^{8}$ C. Giroud, ${ }^{8}$ G. Corrigan, ${ }^{8}$ V. Naulin, ${ }^{9}$ G. Tardini, ${ }^{10}$ K.-D. Zastrow, ${ }^{8}$ and \\ JET-EFDA Contributors ${ }^{\text {a) }}$ \\ JET-EFDA, Culham Science Centre, OX14 3DB Abingdon, United Kingdom \\ ${ }^{1}$ Istituto di Fisica del Plasma, EURATOM/ENEA-CNR Association, 20125 Milano, Italy \\ ${ }^{2}$ VTT, Association EURATOM-Tekes, P.O. Box 1000, FIN-02044 VTT, Finland \\ ${ }^{3}$ Centro de Fusão Nuclear, Associação EURATOM/IST, 1049-001 Lisbon, Portugal \\ ${ }^{4}$ Centre for Fusion Space and Astrophysics, University of Warwick, Coventry 7AL, United Kingdom \\ ${ }^{5}$ Association EURATOM-Tekes, Helsinki University of Technology, P.O. Box 2200, FIN-02150 TKK, Finland \\ ${ }^{6}$ Association Euratom-Hellenic Republic, National Technical University of Athens, \\ GR-15773 Athens, Greece \\ ${ }^{7}$ Euratom-VR Association and Chalmers University of Technology, SE-412 96 Göteborg, Sweden \\ ${ }^{8}$ Euratom/CCFE Association, Culham Science Centre, Abingdon, OX14 3DB, United Kingdom \\ ${ }^{9}$ Association Euratom-Ris $\phi$, DTU, DK-4000 Roskilde, Denmark \\ ${ }^{10}$ Max-Planck-Institut für Plasmaphysik, EURATOM/MPI Association, D-85748 Garching, Germany
}

(Received 12 May 2010; accepted 20 July 2010; published online 28 September 2010)

Perturbative experiments have been carried out in the Joint European Torus [Fusion Sci. Technol. 53(4) (2008)] in order to identify the diffusive and convective components of toroidal momentum transport. The torque source was modulated either by modulating tangential neutral beam power or by modulating in antiphase tangential and normal beams to produce a torque perturbation in the absence of a power perturbation. The resulting periodic perturbation in the toroidal rotation velocity was modeled using time-dependent transport simulations in order to extract empirical profiles of momentum diffusivity and pinch. Details of the experimental technique, data analysis, and modeling are provided. The momentum diffusivity in the core region $(0.2<\rho<0.8)$ was found to be close to the ion heat diffusivity $\left(\chi_{\phi} / \chi_{\mathrm{i}} \sim 0.7-1.7\right)$ and a significant inward momentum convection term, up to $20 \mathrm{~m} / \mathrm{s}$, was found, leading to an effective momentum diffusivity significantly lower than the ion

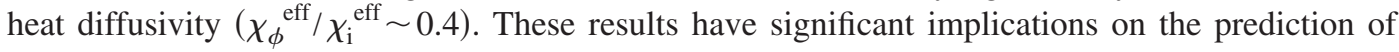
toroidal rotation velocities in future tokamaks and are qualitatively consistent with recent developments in momentum transport theory. Detailed quantitative comparisons with the theoretical predictions of the linear gyrokinetic code GKW [A. G. Peeters et al., Comput. Phys. Commun. 180, 2650 (2009)] and of the quasilinear fluid Weiland model [J. Weiland, Collective Modes in Inhomogeneous Plasmas (IOP, Bristol, 2000)] are presented for two analyzed discharges.

[doi:10.1063/1.3480640]

\section{INTRODUCTION}

The study of plasma rotation and momentum transport in tokamaks is currently experiencing an intensive experimental and theoretical research effort, in view of extrapolating plasma performance from present devices to future machines like ITER. ${ }^{1}$ The role of sheared rotation in the quenching of turbulence with subsequent improvement in confinement $\mathrm{t}^{2-4}$ is well known. In addition, the stabilizing effect of toroidal rotation on pressure limiting resistive wall modes has been pointed out, ${ }^{5}$ although recent developments including kinetic effects have eased the situation on this front. ${ }^{6}$ The lower rotation expected as a consequence of lower torque and larger inertia may thus be detrimental for ITER performance. However, a reliable prediction of magnitude and profile of

\footnotetext{
${ }^{a}$ See Appendix of F. Romanelli et al., Fusion Energy Conference 2008 (Proceedings of the 22nd International Conference, Geneva, 2008), IAEA, Vienna (2008).
}

toroidal rotation in a device like ITER is presently still not possible, both because understanding of momentum transport is still incomplete, although good progress has been achieved recently, and because we lack a precise knowledge of all torque sources and plasma momentum self-generation processes. These reasons motivate further research on momentum transport, in particular experimental investigations against which to validate theoretical models.

The momentum diffusivity $\chi_{\phi}$ and pinch velocity $v_{\text {pinch }}$ (negative sign denotes inward) are related to the toroidal velocity $v_{\phi}$, its gradient $\nabla v_{\phi}$, and the momentum flux $\Gamma_{\phi}$, assuming the absence of a significant particle flux, as follows:

$$
\Gamma_{\phi} \sim-n \chi_{\phi} \nabla v_{\phi}+n \quad v_{\text {pinch }} v_{\phi}=-n \chi_{\phi}^{\text {eff }} \nabla v_{\phi},
$$

where $n$ is the ion density. It is always possible to combine the diffusive and convective parts of the momentum flux into an effective momentum diffusivity $\chi_{\phi}^{\text {eff }}$, which can be calculated from steady-state rotation profiles once momentum sources are known. In the following the term "steady-state" 
will be used to indicate a quantity taken at a given time (or time-averaged over a given interval), therefore not resolving the modulation time dependence, while the term "stationary" will be used to indicate conditions where plasma parameters and profiles do not exhibit significant slow trends.

On the theoretical side, substantial progress has been achieved in the understanding of momentum transport driven by ion temperature gradient (ITG) modes. ${ }^{7-12}$ Fluid models and gyrokinetic codes have been developed, which link inherently momentum and ion heat transport, foreseeing similar diffusivities for both channels (Prandtl number, $\left.P_{\mathrm{r}}=\chi_{\phi} / \chi_{\mathrm{i}} \sim 1\right)$. In addition, the recently predicted existence of an inward momentum pinch is a qualitatively new ingredient in assessing the achievable rotation peaking in ITER, where torque sources will be located mainly in the outer regions. As found for particle transport, the impact of the pinch existence on the plasma profiles would be higher for momentum than for heat, for which the presence of an inward pinch, if any, is always dominated by the high diffusive transport component due to the core localization of heat sources.

On the experimental side, a significant improvement in the characterization of the steady-state behavior of toroidal rotation has been achieved, both in plasmas with external torque source ${ }^{13-16}$ and in plasmas without external torque sources, ${ }^{17,18}$ which still exhibit nonzero toroidal rotation, the so-called "intrinsic rotation," whose physical origin is still under investigation. A rather surprising recent observation from steady-state momentum studies is that the effective momentum diffusivity is much lower than the ion heat diffusivity $\left[P_{\mathrm{r}}^{\text {eff }}=\chi_{\phi}{ }^{\text {eff }} / \chi_{\mathrm{i}}^{\text {eff }} \sim 0.4\right.$ using the definition of $\chi_{\phi}$, as in Eq. (1)], ${ }^{13,15,16}$ apparently contradicting the theoretical expectations. It is clear that the existence of a momentum inward pinch would solve the apparent contradiction, allowing for similar diagonal diffusivities at the same time as different effective values. However, a direct demonstration of the existence of a momentum pinch is fairly difficult from steadystate data only. A clean way of identifying separately diffusive and convective transport components is by means of perturbative experiments, ${ }^{19}$ i.e., exploiting the additional information contained in the dynamic response of the plasma rotation to a time variation of the torque source. Modulation at a suitable frequency has the advantage of optimizing the $\mathrm{S} / \mathrm{N}$ ratio by averaging over several cycles and has been used successfully to study electron and more recently ion heat transport, and also particle and impurity transport (see Ref. 20 for a recent review). Its application to momentum transport studies using neutral beam injection (NBI) modulation is a recent development, with a couple of studies reported in JT-60U, ${ }^{21,22}$ one experiment reported in the Joint European Torus (JET) (Refs. 23-25), and one in DIII-D. ${ }^{26}$ The use of magnetic perturbations to brake the plasma was reported in DIII-D (Ref. 27) and in the National Spherical Torus Experiment (NSTX) (Refs. 26, 28, and 29) as another means to induce a transient from which it is possible to infer diffusivity and convection separately. In addition, DIII-D has the unique capability of being able to zero out applied torque at constant power.
This paper describes momentum perturbative experiments using NBI modulation in the JET tokamak, ${ }^{50}$ presenting both new experiments and more refined analysis and modeling with respect to previous work ${ }^{23-25}$ and including detailed comparison of the results with recent gyrokinetic and fluid theory predictions. Section II illustrates the experiments, Sec. III discusses the calculations of the timedependent torque deposition, Sec. IV illustrates the rather heavy experimental methodology, based on transport modeling required to extract from the data the estimates of momentum diffusivity and pinch, Sec. V summarizes the linear gyrokinetic predictions, Sec. VI describes first attempts to perform time-dependent modeling of the rotation dynamic response with 1D quasilinear fluid transport models. Section VII summarizes the results and discusses future developments.

\section{EXPERIMENTAL RESULTS}

\section{A. Outlook of experimental set-up}

The toroidal momentum perturbative experiments have been carried out in low collisionality JET H-mode plasmas $\left(B_{\mathrm{T}}=3 \mathrm{~T}, I_{\mathrm{p}}=1.5 \mathrm{MA}, n_{\mathrm{e} 0} \sim 4 \times 10^{19} \mathrm{~m}^{-3}, \quad \beta_{\mathrm{n}}=0.4-0.5\right.$, $\left.\nu^{*}=0.01-0.015, P / P_{\mathrm{LH}} \sim 2-3\right)$ with minimum level of magnetohydrodynamic activity to prevent interference with the perturbation analysis, i.e., at high $q_{95} \sim 7$, to avoid large sawteeth in the center, and with type III edge localized modes, to avoid large periodic edge crashes without changing the core transport mechanisms. Total power levels were up to $13 \mathrm{MW}$ for NBI and $4 \mathrm{MW}$ for ion cyclotron resonance heating in $\mathrm{H}$ minority scheme. In these conditions, ITGs are the dominant instability, making the coupling of momentum and ion heat transport, and thus the concept of Prandtl number, unambiguous. The NBI torque source is the best available tool on JET for inducing a significant rotation perturbation, although it is not ideal because its deposition profile is very broad, which is a complicating factor in the analysis, unlike in the case of localized rf heat sources. On the other hand, the torque sources from NBI can be calculated more precisely than radio-frequency power sources. Momentum transfer from the NBI fast ions to the thermal bulk plasma takes place via two main mechanisms: ${ }^{30}$ (a) passing ions transfer toroidal angular momentum to the bulk plasma by collisions, which is a slow process; (b) trapped ions transfer their momentum by $\boldsymbol{J} \times \boldsymbol{B}$ forces, due to the (radial) displacement current that sets in to ensure charge neutrality following the ion motion along a banana orbit of finite width. This is practically an instantaneous process $(\boldsymbol{J}$ denotes the displacement current density and $\boldsymbol{B}$ the magnetic field). The collisional torque dominates in the center while the $\boldsymbol{J} \times \boldsymbol{B}$ torque dominates from midradius to the plasma edge. If the frequency of the modulation is fast enough compared to the fast ion slowing down time, the $\boldsymbol{J} \times \boldsymbol{B}$ component will dominate over the collisional one in the perturbation source. In the analysis both collisional and $\boldsymbol{J} \times \boldsymbol{B}$ torque have been taken into account. In the JET experiments, the NBI power and torque were square wave modulated with a duty cycle $\mathrm{dc}=50 \%$ or $33 \%$ at a frequency $f=6.25$ or $8.33 \mathrm{~Hz}$, which is the highest technically possible. Using the latter, $\boldsymbol{J} \times \boldsymbol{B}$ torque becomes the 


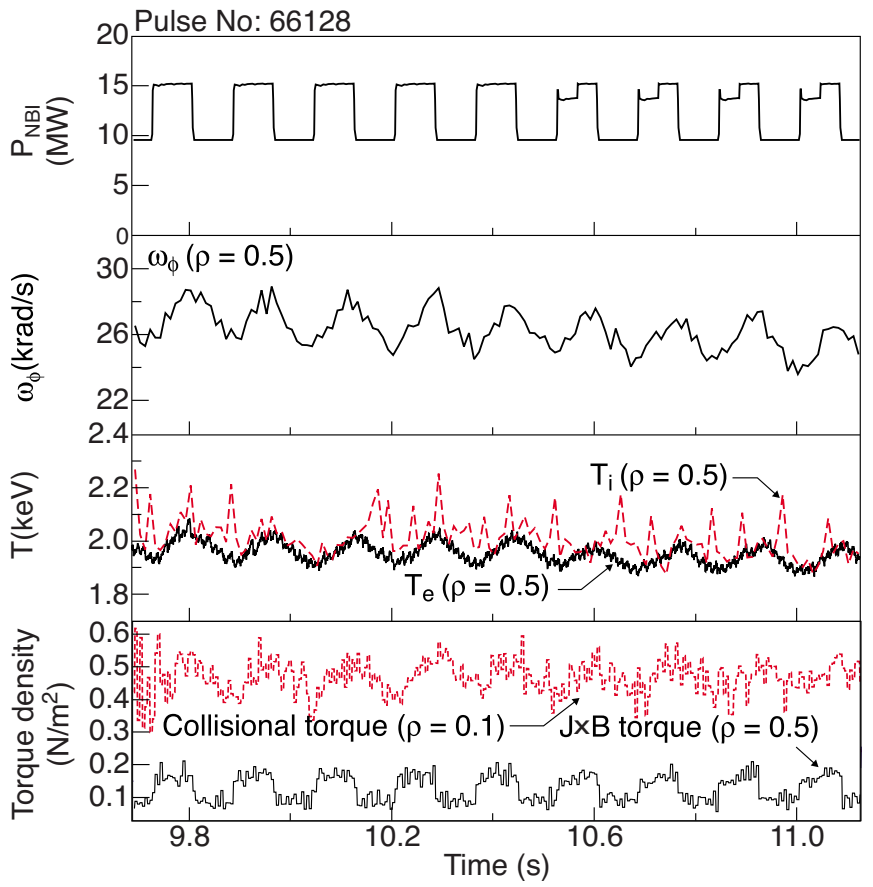

FIG. 1. (Color online) Time traces of the modulated NBI power, $\omega_{\phi}, T_{\mathrm{i}}$, and $T_{\mathrm{e}}$ and the two different components of the torque density for JET shot 66128 (noncompensated case).

dominant source of torque perturbation. The modulation frequency is much slower than the $10 \mathrm{~ms}$ time resolution of the charge exchange recombination spectroscopy (CXRS) diagnostic used to measure the toroidal rotation profile $\omega_{\phi}$ and ion temperature $T_{\mathrm{i}}$ at 12 radial points, with an uncertainty of $\pm 5 \%$ on their absolute values and $\sim \pm 10 \%$ on their gradients. ${ }^{31}$ Consequently, it is possible to observe changes in toroidal rotation in the beam on and beam off periods and perform the Fourier analysis of the modulated rotation. The modulation took place in a stationary phase for several seconds following two basic schemes:

(1) Torque modulation with noncompensated power modulation, modulating $(80 \mathrm{~ms}$ on, $80 \mathrm{~ms}$ off or $40 \mathrm{~ms}$ on, $80 \mathrm{~ms}$ off) 3-4 tangential beams up to $6 \mathrm{MW}$ of modulated power. This technique is the most straightforward and allows a good $\mathrm{S} / \mathrm{N}$ level in the toroidal rotation modulation. As discussed above, the perturbed torque source is the sum of the collisional and $\boldsymbol{J} \times \boldsymbol{B}$ torque delivered by each tangential beam. Time traces of NBI power, torque density at two radial locations as calculated by TRANSP (Ref. 32) (see Sec. III), toroidal angular rotation frequency $\omega_{\phi}=v_{\phi} / R$ ( $R$ is the major radius), and $T_{\mathrm{i}}$ and $T_{\mathrm{e}}$ at midradius for nine of the modulation cycles are illustrated in Fig. 1. Steady-state profiles of $n_{\mathrm{e}}, T_{\mathrm{e}}, T_{\mathrm{i}}, \omega_{\phi}$, and TRANSP calculated torque density are shown in Fig. 2. All spatial information in the paper is provided using as radial coordinate the normalized toroidal radius $\rho_{\text {tor }}=\rho_{\mathrm{t}} / \rho_{\mathrm{t}}^{\text {edge }}$, where $\rho_{\mathrm{t}}=\sqrt{ } \Phi / \pi B_{0}$, with $\Phi$ the toroidal magnetic flux, and $\rho_{\mathrm{t}}^{\text {edge }}$ is the value of $\rho_{\mathrm{t}}$ at the plasma boundary. One can see in Fig. 1 the different time behavior of the $\boldsymbol{J} \times \boldsymbol{B}$ and collisional torque, the first having the same square wave

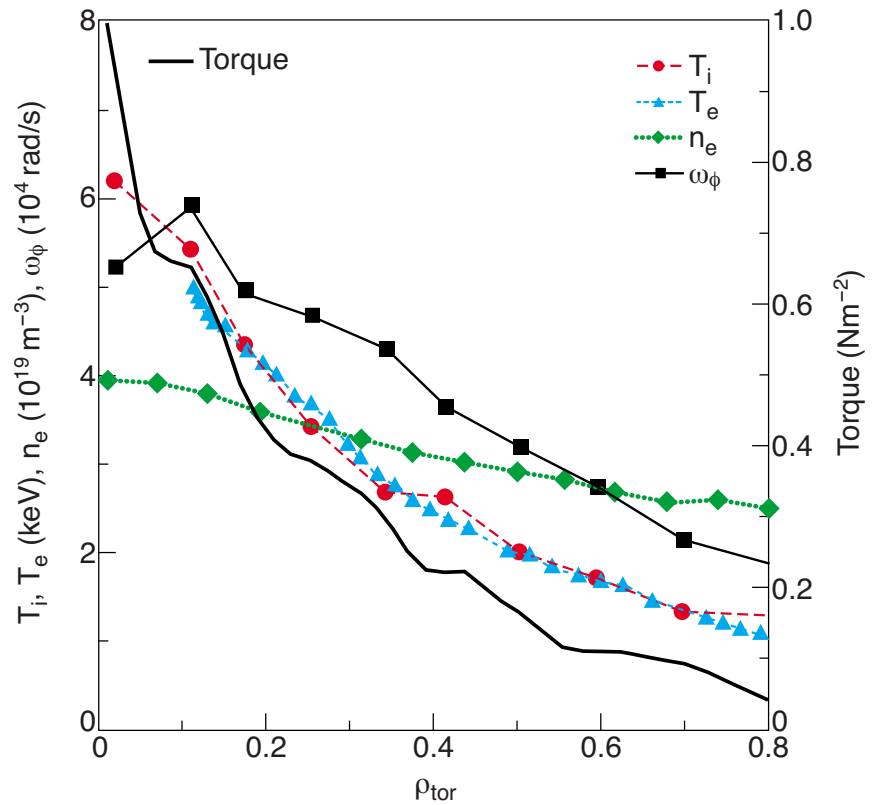

FIG. 2. (Color online) Steady-state radial profiles at $t=10 \mathrm{~s}$ of $\omega_{\phi}, T_{\mathrm{i}}, T_{\mathrm{e}}$, $n_{\mathrm{e}}$, and torque density for JET shot 66128 .

modulation as power, due to instantaneous deposition, the second being integrated by the fast ion slowing down time. A clear rotation modulation is visible in Fig. 1. Due to the concomitant power modulation, also the electron and ion temperature are modulated, as well as the total energy content and plasma position, which are complications to be accounted for in the analysis, as discussed in Secs. II B and IV A 2.

(2) Torque modulation with compensated power modulation, modulating in antiphase three tangential and three normal beams (50 ms on tangential, $110 \mathrm{~ms}$ on normal). In this way there is no net power modulation, hence no temperature and plasma position modulation. The torque modulation also partly cancels, the remaining torque modulated component being due to the differences in torque deposition between tangential and normal beams. While beam particles absorbed at the high field side of magnetic axis become passing ions, those absorbed at the low field side become either passing or trapped ions depending on the inverse aspect ratio of the flux surface where they are created, $\varepsilon=r / R$. For the parameters of the so-called normal neutral beams on JET, the fast ions are passing, i.e., $\xi=R_{\text {imp }} / R>\xi_{t}=\sqrt{2 \varepsilon / 1+\varepsilon}$ (where $R_{\text {imp }}$ is the impact radius), for $\rho_{\text {tor }}<0.24$, while for the tangential neutral beam bank they are passing for $\rho_{\text {tor }}<0.39$. Therefore in a simplified picture, in the inner plasma region $0.24<\rho_{\text {tor }}<0.39$ the perturbed source will be dominated by the normal beams, while in an intermediate region outside $\rho_{\text {tor }}=0.39$ there would be cancellation of the $\boldsymbol{J} \times \boldsymbol{B}$ component of normal and tangential beams, leaving further out a $\boldsymbol{J} \times \boldsymbol{B}$ component from tangential beams. The collisional torque component from tangential and normal beams should also to large extent cancel, decreasing its weight in comparison with the noncompensated case. Therefore this experi- 


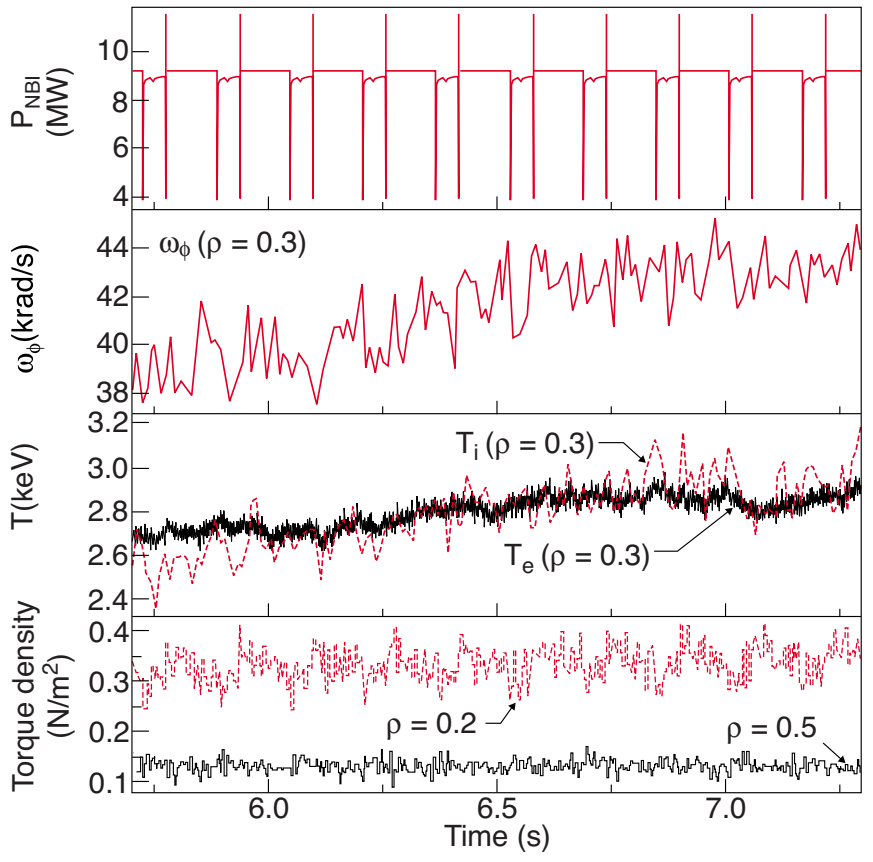

FIG. 3. (Color online) Time traces of the total NBI power, $\omega_{\phi}, T_{\mathrm{i}}$, and $T_{\mathrm{e}}$ at $\rho=0.3$ and the total torque density at $\rho=0.2$ and $\rho=0.5$ for JET shot 73700 (compensated case).

ment should have resulted in a better spatial localization of the perturbed torque source, although with somewhat peculiar phasing, possibly allowing transport analysis in a source free region. However, as discussed more in detail in Sec. III on the basis of the Fourier analysis of the TRANSP calculated torque, the actual torque cancellation was not complete, and in addition also the $\mathrm{S} / \mathrm{N}$ of the rotation perturbation in these experiments turned out much lower, as can be seen from the time traces in Fig. 3 (Fourier analysis is the only way to detect the rotation

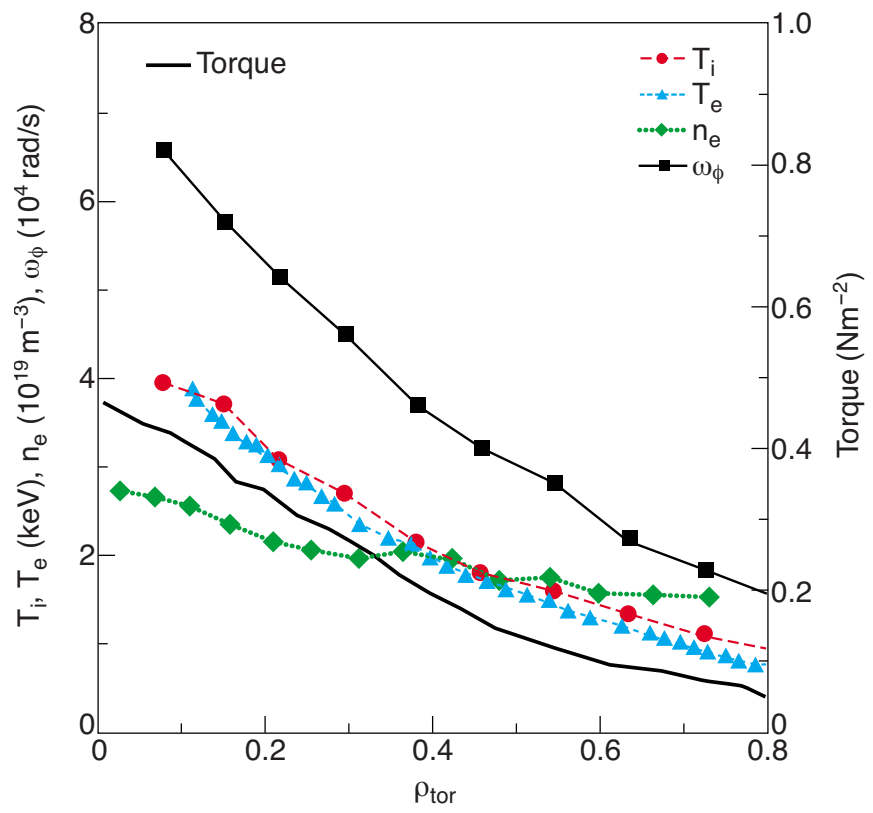

FIG. 4. (Color online) Steady-state radial profiles at $t=6.1 \mathrm{~s}$ of $\omega_{\phi}, T_{\mathrm{i}}, T_{\mathrm{e}}$, $n_{\mathrm{e}}$, and torque density for JET shot 73700 . modulation). Figure 4 shows the steady-state profiles for this case. In conclusion this configuration did not deliver the anticipated advantages in spite of the additional complications of the experiment and has therefore been abandoned in our later experiments on JET.

\section{B. Fourier analysis of rotation and temperature modulation and corrections for oscillating plasma position}

Standard fast Fourier transform has been applied to the experimental time traces at various radial positions of $\omega_{\phi}, T_{\mathrm{i}}$, and $T_{\mathrm{e}}$, to derive spatial profiles of amplitude $(A)$ and phase $(\varphi)$ of the perturbation at the first harmonic of the modulation frequency for $\mathrm{dc}=50 \%$ and also at the second harmonic for $\mathrm{dc}=33 \%$. The third harmonic is always below noise level. Using two harmonics improves the separate identification of the diffusive and convective components of the momentum flux, since the impact of the momentum pinch on the modulated data vanishes at high frequency. ${ }^{19}$ The profiles are shown in Fig. 5 for a noncompensated case at $6.25 \mathrm{~Hz}$, $50 \%$ dc, in Fig. 6 for a similar shot with noncompensated modulation at $8.33 \mathrm{~Hz}, 33 \% \mathrm{dc}$, and in Fig. 7 for a compensated case at $6.25 \mathrm{~Hz}, 33 \%$ dc. For the first noncompensated case also the temperature perturbations are shown, while for the compensated case the temperature perturbation is null. Phase values are always calculated with respect to the phase of the tangential beam power. The statistical error bars are calculated for the amplitudes from the noise level outside the spectral peaks and for the phases by assuming that the noise level would add up to the signal with a $90^{\circ}$ phase shift (worst case).

As mentioned in Sec. II A, in the noncompensated case the plasma equilibrium changes periodically with the modulated NBI power. This is important as the experimental CXRS data are measured at fixed positions in the laboratory frame, resulting in a spurious measured $\omega_{\phi}, T_{\mathrm{i}}$, and $T_{\mathrm{e}}$ oscillation given by the product of their gradient and displacement (as a complex number). This would falsify the transport analysis if not properly taken into account. There is both a rigid displacement of the whole plasma and a modulation of the Shafranov shift which affects only the core. They are comparable in magnitude, each in the order of $\pm 5 \mathrm{~mm}$ for the $5 \mathrm{MW}$ NBI modulation in the low current plasmas used for these experiments. Rotation and temperature must therefore be properly mapped into a plasma movement independent radial coordinate system before performing the transport analysis and modeling. Here, this is done within TRANSP using the time-dependent EFIT (Ref. 33) reconstructed equilibrium constrained by motional Stark effect measurements to map all experimental data onto the same flux surface grid used for the torque calculation and the transport simulations. Both horizontal and vertical plasma oscillations are taken into account. The outcome of the correction procedure is illustrated in detail in Figs. 5(a) and 5(b), where the corrected $A$ and $\varphi$ profiles are shown by dashed lines and compared to uncorrected values in Fig. 5(a) for $\omega_{\phi}$ and in Fig. 5(b) for $T_{\mathrm{i}}$. 


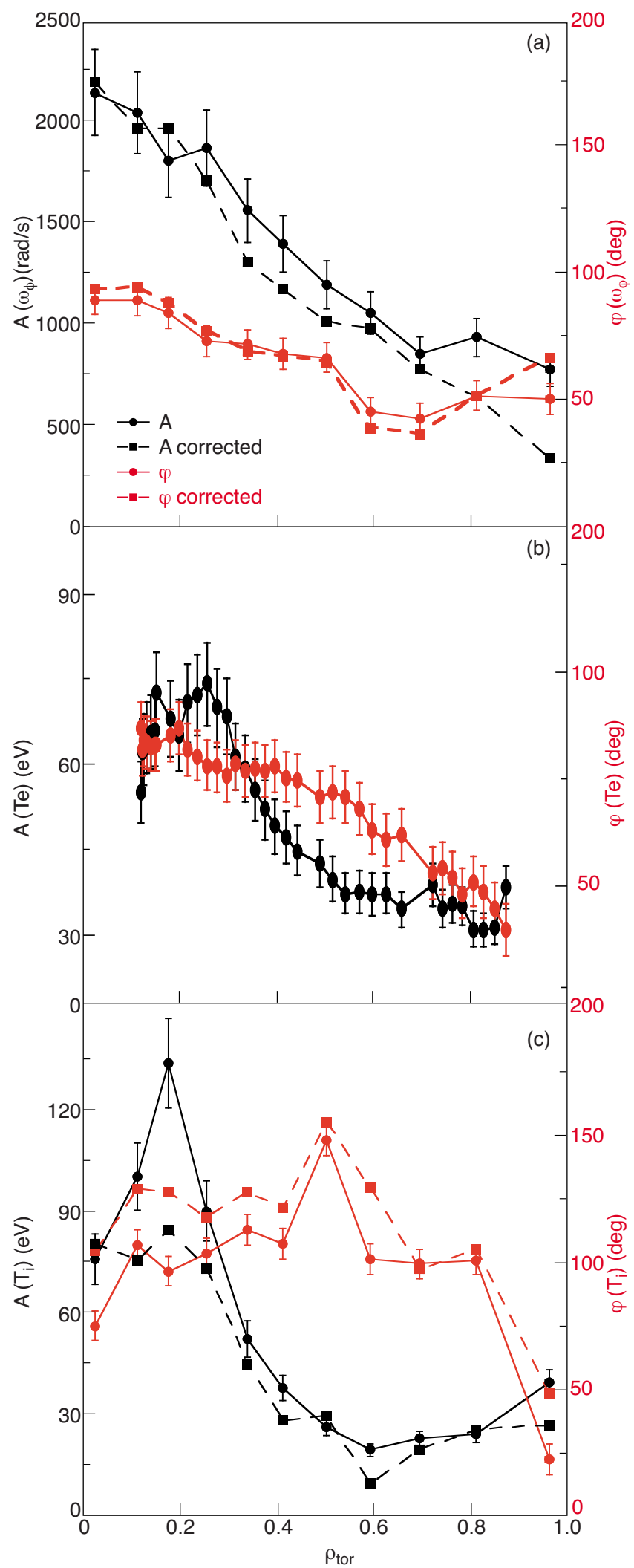

FIG. 5. (Color online) Radial profiles of $A$ and $\varphi$ at $6.25 \mathrm{~Hz}$ in shot 66128 for (a) $\omega_{\phi}$, (b) $T_{\mathrm{e}}$, and (c) $T_{\mathrm{i}}$. In (a) and (c) the data corrected for oscillating plasma position are shown.

The details of $T_{\mathrm{e}}$ modulation are not important for the following analysis and modeling, so only the uncorrected data are shown for the sake of information. The effect of oscillating displacement is found rather important on core $T_{\mathrm{i}}$ oscil-

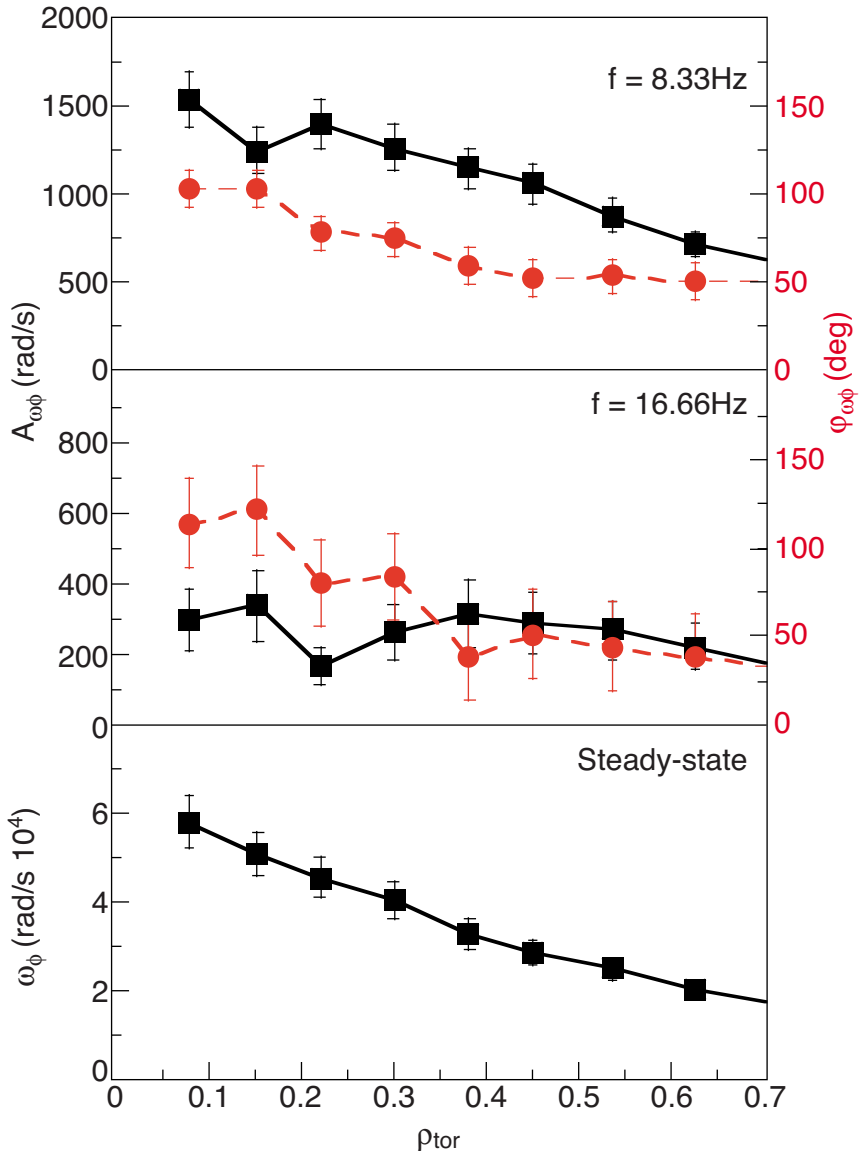

FIG. 6. (Color online) Radial profiles of $A$ (black squares) and $\varphi$ (red circles) at 8.33 and $16.66 \mathrm{~Hz}$ and steady-state $\omega_{\phi}$ in shot 73701 .

lations but minor on $\omega_{\phi}$ oscillations. This is due to the higher peaking of the $T_{\mathrm{i}}$ profile compared to the $\omega_{\phi}$ profile. The data in Fig. 6 are already corrected. This correction is obviously not needed in the compensated case.

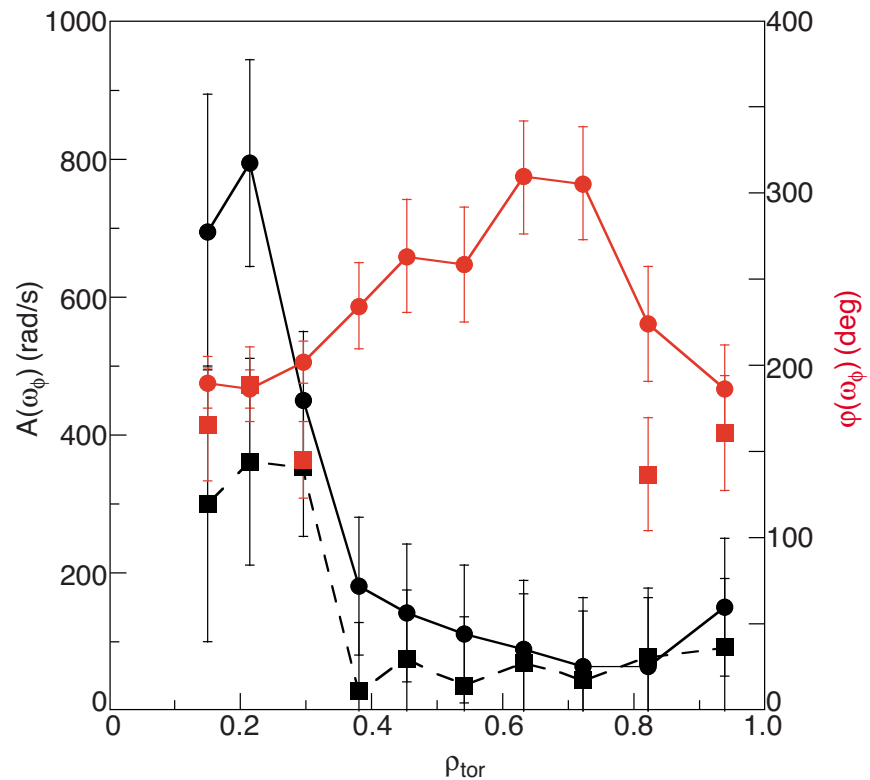

FIG. 7. (Color online) Radial profiles of $A$ (black) and $\varphi$ [red (grey in print)] of $\omega_{\phi}$ at $6.25 \mathrm{~Hz}$ (circles) and $12.50 \mathrm{~Hz}$ (squares) in shot 73700 (compensated case). Phase values are not shown for second harmonic at locations where amplitudes are below noise level. 
It can be noted in Fig. 5(a) that the rotation perturbation $A$ and $\varphi$ profiles are highly influenced by the nonlocalized source profiles, made by two different components with a time shift, rather than by transport processes only, at variance with most perturbative heat transport experiments. In fact the amplitude is maximum in the center, corresponding to the collisional torque source which is dominant there at $6.25 \mathrm{~Hz}$, but the phase is minimum around $\rho \sim 0.6$, which is the region where the collisional torque has vanished and only the instantaneous $\boldsymbol{J} \times \boldsymbol{B}$ torque is acting. The increase in $\varphi$ toward the center is not only due to propagation of the $\boldsymbol{J} \times \boldsymbol{B}$ rotation wave, but also to the high phase values associated to the fast ion slowing down that provides the central collisional torque source. This is a clear indication that, due to the nonlocalization of the modulated torque source, a simple determination of the momentum diffusivity and pinch directly from the spatial derivatives of the amplitude and phase of the modulated $\omega_{\phi}$ is not viable. Therefore, time-dependent transport modeling of $\omega_{\phi}$ is required to extract the transport properties from the plasma dynamic response, assuming that torque sources are known with reasonable accuracy from numerical calculations.

Figure 7 illustrates the measured $A$ and $\varphi$ for the compensated experiment. The results are in line with the expectation of a much narrower torque source in the center, since at midradius $\left(0.3<\rho_{\text {tor }}<0.7\right)$, there is a zone of partial cancellation between normal and tangential beams. However, a priori we would have expected a much clearer separation of normal and tangential peaks in the amplitude profiles (see discussion on torque source in Sec. III A). The transition from normal to tangential torque component is clearly visible in the large increase of phase between $\rho_{\text {tor }}=0.2$ (where normal beams dominate) and $\rho_{\text {tor }}=0.7$ (where tangential beams dominate, although with much smaller amplitudes), due to the $180^{\circ}$ phase jump in the torque of the two sources. The very external region is again dominated by normal beams, as can be seen from the phase values in the region $\rho_{\text {tor }}>0.8$. This could be due to a larger deposition of trapped particles in the external region by the normal beams. This region $\left(\rho_{\text {tor }}>0.8\right)$ is outside the scope of this paper which focuses on core momentum transport. Also TRANSP torque calculations become less reliable in this region where the plasma profiles are not known with similar accuracy as in the core.

\section{NUMERICAL COMPUTATION \\ OF THE TIME-DEPENDENT TORQUE SOURCE}

\section{A. Power and torque calculations using TRANSP and ASCOT}

Due to the absence of a source free region where the transport analysis could be done independently of the details of the source, the calculation of the time-dependent torque profile is an essential step for the derivation of the momentum transport coefficients from the data. The transport analysis will in fact rely on full transport simulations in which the source is input as known, with the obvious consequence that any error in the source will determine an error on the derived transport coefficients. Great care has then been dedicated to such torque calculations and associated uncertainties. In the following we will consider the NBI as the only torque source, since residual stress or other intrinsic sources are definitely negligible in the presence of 10-15 MW NBI power, as discussed in Ref. 25.

The TRANSP code ${ }^{32}$ has been used to calculate NBI power and torque profiles as a function of time, given the time-dependent experimental profiles of all plasma parameters, but neglecting the small temporal oscillations of plasma parameters due to the NBI modulation itself, to avoid polluting with noise the main torque oscillation coming from the NBI oscillating waveform. $T_{\mathrm{i}}$ has been taken from CXRS, $T_{\mathrm{e}}$ from electron cyclotron emission radiometer and light detection and ranging (LIDAR), $n_{\mathrm{e}}$ from interferometer and LIDAR, $Z_{\text {eff }}$ from CXRS and spectroscopic measurements $\left(Z_{\text {eff }} \sim 2\right.$ in these discharges, a radially constant profile has been assumed). Error bars are typically $\pm 5 \%$ for $T_{\mathrm{i}}$ and $T_{\mathrm{e}}, \pm 10 \%$ for $n_{\mathrm{e}}, \pm 20 \%$ for $Z_{\text {eff. }}$. The internal TRANSP equilibrium solver with EFIT data as boundary condition was used to calculate equilibrium consistently with the slow time variations of profiles. The fast equilibrium variations due to modulation have been neglected because as discussed in Sec. II B their effect has been removed from the data before they are compared with simulations. TRANSP contains the NUBEAM Monte Carlo solver, ${ }^{34}$ which has always been used with a high number $(160000)$ of particles. A test of the sensitivity of torque amplitudes and phases to the number of Monte Carlo particles has shown that in excess of 80000 particles are needed to reach convergence in the results. The torque calculations are reliable up to $\rho_{\text {tor }}=0.8$, due to uncertainties in the plasma parameters in the external region.

Figure 8 shows $A$ and $\varphi$ of the NBI total torque density and of the ion and electron power densities calculated for shot 66128. The steady-state torque density for this shot was already shown in Fig. 2. The central torque density perturbation is typically in the order of $10 \%$ while the central power density perturbation is $\sim 3 \%$. One can notice the zero-phase, instantaneous torque deposition for $\rho_{\text {tor }}>0.4$ due to the $\boldsymbol{J} \times \boldsymbol{B}$ torque, while in the central region the main torque component is the delayed collisional one. The ion and electron power phases also indicate collisional deposition from fast NBI ions to thermal population, with longer collisional times to ions than to electrons.

Figure 9 shows $A$ and $\varphi$ of the calculated torque density for shot 73701, at two harmonics of the modulation frequency, indicating separately the $\boldsymbol{J} \times \boldsymbol{B}$ and collisional components. In this shot due to higher modulation frequency ( $8.33 \mathrm{~Hz}$ rather than $6.25 \mathrm{~Hz}$ of shot 66128 ), the $\boldsymbol{J} \times \boldsymbol{B}$ torque dominates at all radii but the very central region.

In order to gain confidence in the correctness of the TRANSP NUBEAM calculations, the modulated torque for 73701 has been calculated also with the orbit following ASCOT code. $^{35}$ ASCOT is similar to NUBEAM module within TRANSP. Both codes are guiding center following Monte Carlo codes with internal NBI birth profile generator. In ASCOT calculations the small temporal variations in equilibrium due to the NBI modulation are neglected and constant EFIT reconstructed equilibria with motional Stark effect (MSE) constraint are used together with time-averaged plasma profiles. Time varying NBI waveforms provide the 

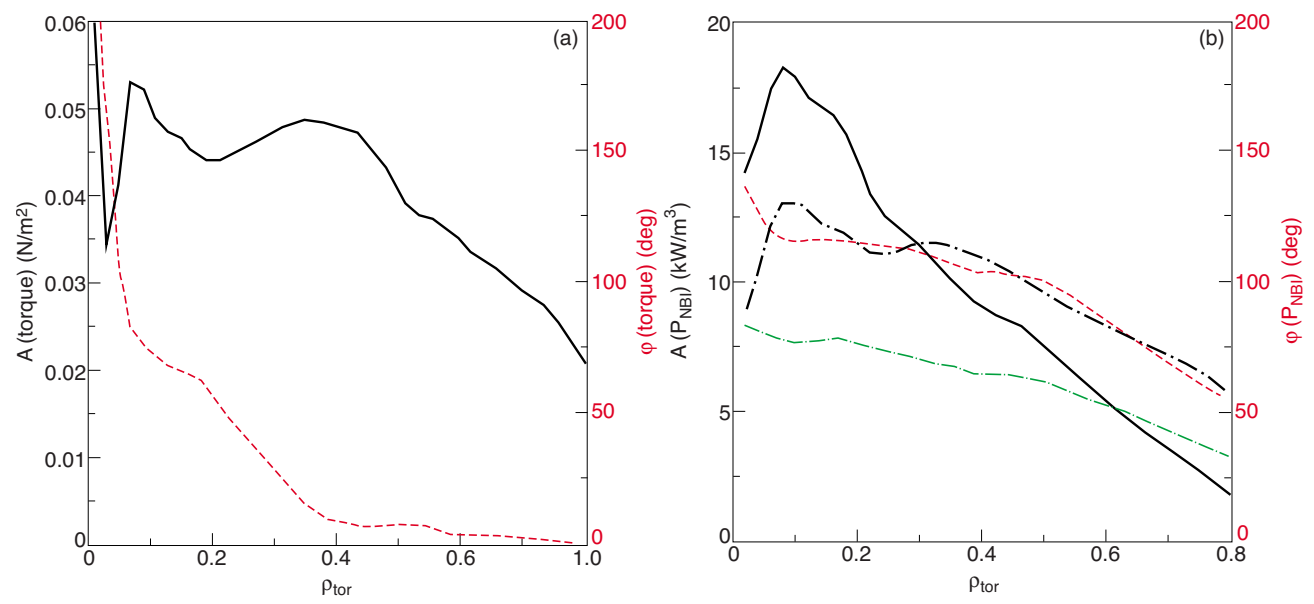

FIG. 8. (Color online) Radial profiles of $A$ and $\varphi$ at $6.25 \mathrm{~Hz}$ in shot 66128 (noncompensated) for TRANSP calculated: (a) total torque density (black full line: $A$, red dashed line: $\varphi$ ); (b) ion power density (black full line: $A$, red dashed line: $\varphi$ ) and electron power density (gray dashed-dotted line: $A$, green dashed line: $\varphi)$.

dynamics in torque profiles. The results for $A$ and $\varphi$ are shown in Fig. 10, again indicating the $\boldsymbol{J} \times \boldsymbol{B}$ and collisional components. Figure 11 compares the steady-state torque density calculated with TRANSP and ASCOT. The agreement between the two very different and independent codes is re- markable and constitutes the most powerful confirmation that we can rely on the torque calculations for our transport analysis.

Finally, the $A$ and $\varphi$ of modulated torque density for the compensated case, shot 73700, are shown in Fig. 12 calcu-

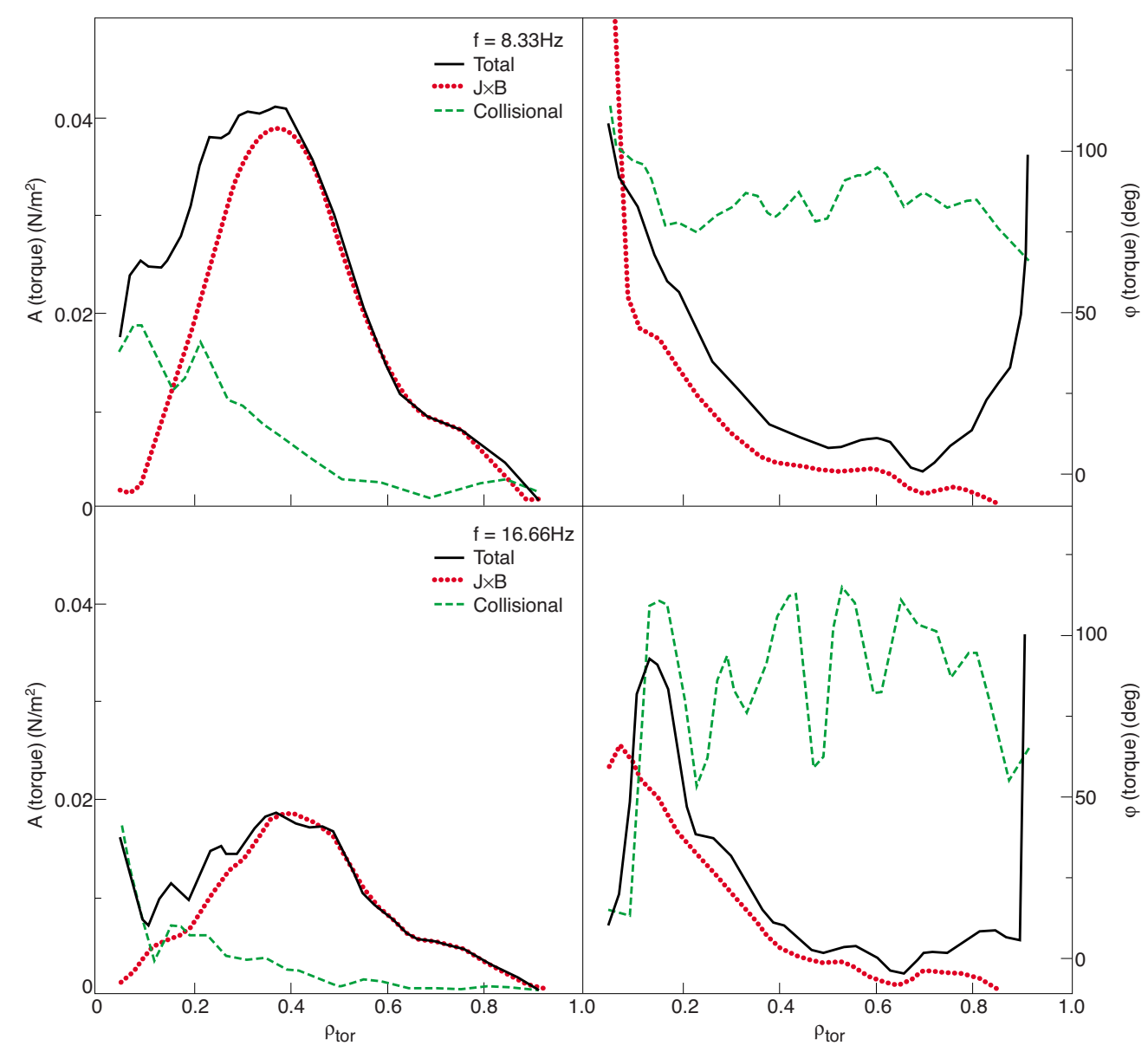

FIG. 9. (Color online) Radial profiles of $A$ and $\varphi$ of TRANSP calculated torque density at 8.33 and $16.66 \mathrm{~Hz}$ in shot 73701 (noncompensated), distinguishing collisional and $\boldsymbol{J} \times \boldsymbol{B}$ components. 


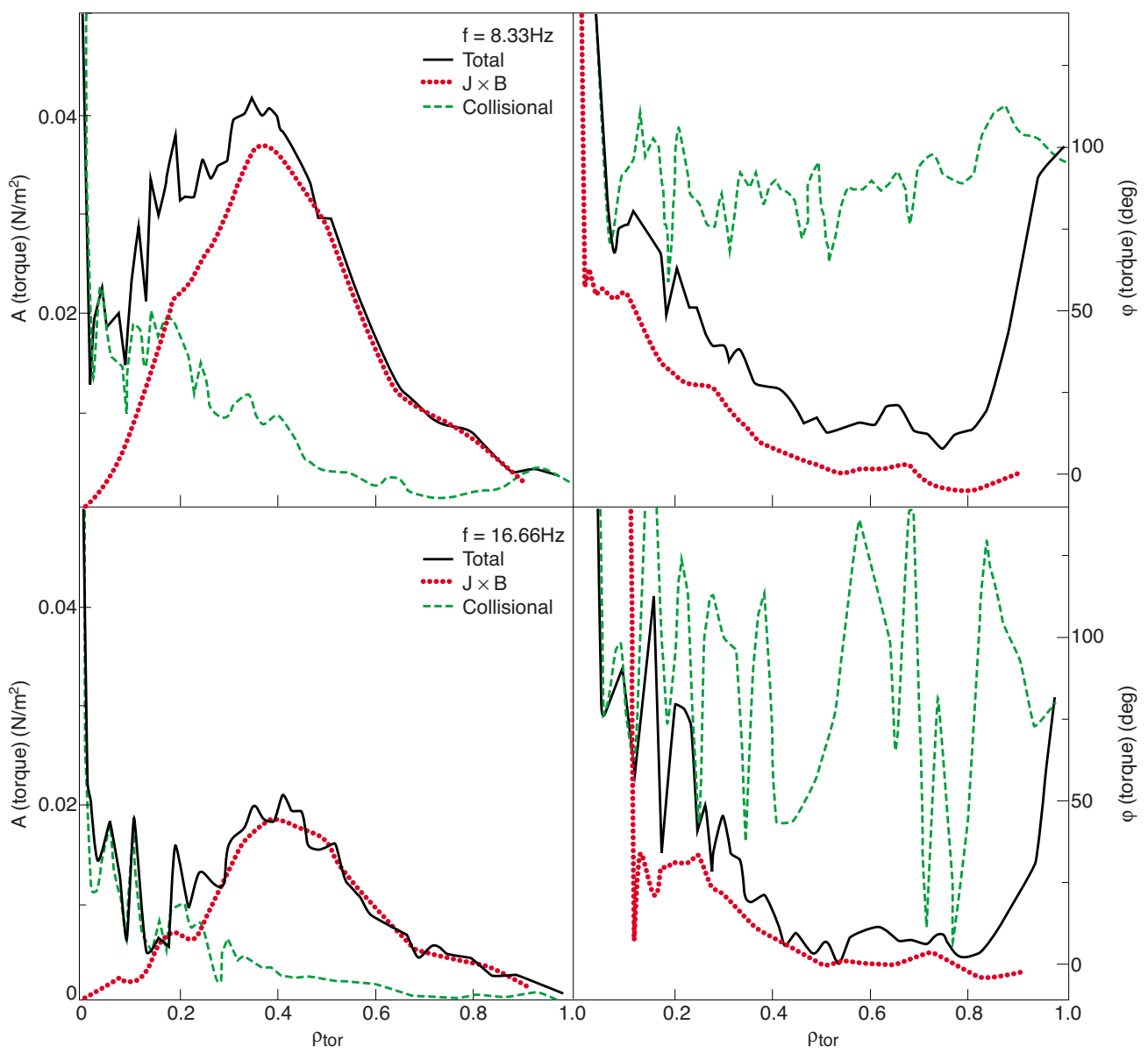

FIG. 10. (Color online) Radial profiles of $A$ and $\varphi$ of ASCOT calculated torque density at 8.33 and $16.66 \mathrm{~Hz}$ in shot 73701 (noncompensated), distinguishing collisional and $\boldsymbol{J} \times \boldsymbol{B}$ components.

lated with TRANSP and in Fig. 13 calculated with ASCOT, and indicating separately the $\boldsymbol{J} \times \boldsymbol{B}$ and collisional components. To help visualization of the complex torque dynamics, in Fig. 14 we also show the $A$ and $\varphi$ of the torque density calculated with ASCOT indicating separately the normal and tangential beams. The phase is always taken with respect to the tangential beams. One can see that the peak in $A$ of the perpendicular beams is shifted slightly inside with respect to the one of the tangential beams, and the two are in phase opposition, so that there is a partial cancellation leading to a more narrow total deposition (with respect to the noncompensated case) dominated by the perpendicular beams in the central region. However at the frequency used $(6.25 \mathrm{~Hz})$ there is still a significant central collisional component that comes mainly from the tangential beams. Moreover, the fact that the cancellation in the external region is not perfect leads to the impossibility of considering such region as source free for the analysis, which in practice nullifies the usefulness of this approach, as will be discussed in detail in Sec. IV B.

\section{B. Analysis of sensitivity to plasma parameters}

Having assessed by comparing two different codes the reliability of the torque calculations for a given set of plasma parameters and profiles, in this section we deal with the assessment of uncertainties in torque following from uncertain- ties in plasma parameters. We have evaluated the impact of the most important players, i.e., $n_{\mathrm{e}}, T_{\mathrm{e}}, Z_{\text {eff }}$. The $\boldsymbol{J} \times \boldsymbol{B}$ torque is essentially instantaneous and depends only on the initial deposition (governed by $n_{\mathrm{e}}$ and $Z_{\text {eff }}$ to lesser extent). $T_{\mathrm{e}}$ and $Z_{\text {eff }}$ affect the slowing down rate and ion to electron heating ratio and thus the torque deposition dynamics. $T_{\mathrm{i}}$ is not a very sensitive player as it has practically no role in the initial deposition and only affects the ion slowing down at low energies, but has no effect on electron slowing down and thus its overall effect on torque dynamics is usually small. Of course, additionally, NBI acceleration voltage and beam fractions play a role but these can be assumed to be known.

Figure 15 shows the variation of $A$ and $\varphi$ of the torque density when (a) $n_{\mathrm{e}}$ is varied between 0.8 and 1.2 of the experimental value, (b) $T_{\mathrm{e}}$ is varied between 0.8 and 1.2 of the experimental value, (c) $Z_{\text {eff }}$ is decreased from the experimental value of 1.9 to 1 . Once can see that the impact is only on amplitudes, not on phases. As we will discuss in Sec. IV A 1, this implies that the momentum diffusivity, or Prandtl number, is a robust quantity, insensitive to errors in plasma parameters. The pinch value, instead, depends on the torque amplitudes and therefore its determination will be affected by larger uncertainties, reflecting those in the measured plasma parameters. Among these, the main impact is given by density, with a variation of about $\pm 15 \%$ when $n_{\mathrm{e}}$ is varied in an interval of $\pm 20 \%$ of the measured value. $T_{\mathrm{e}}$ and 

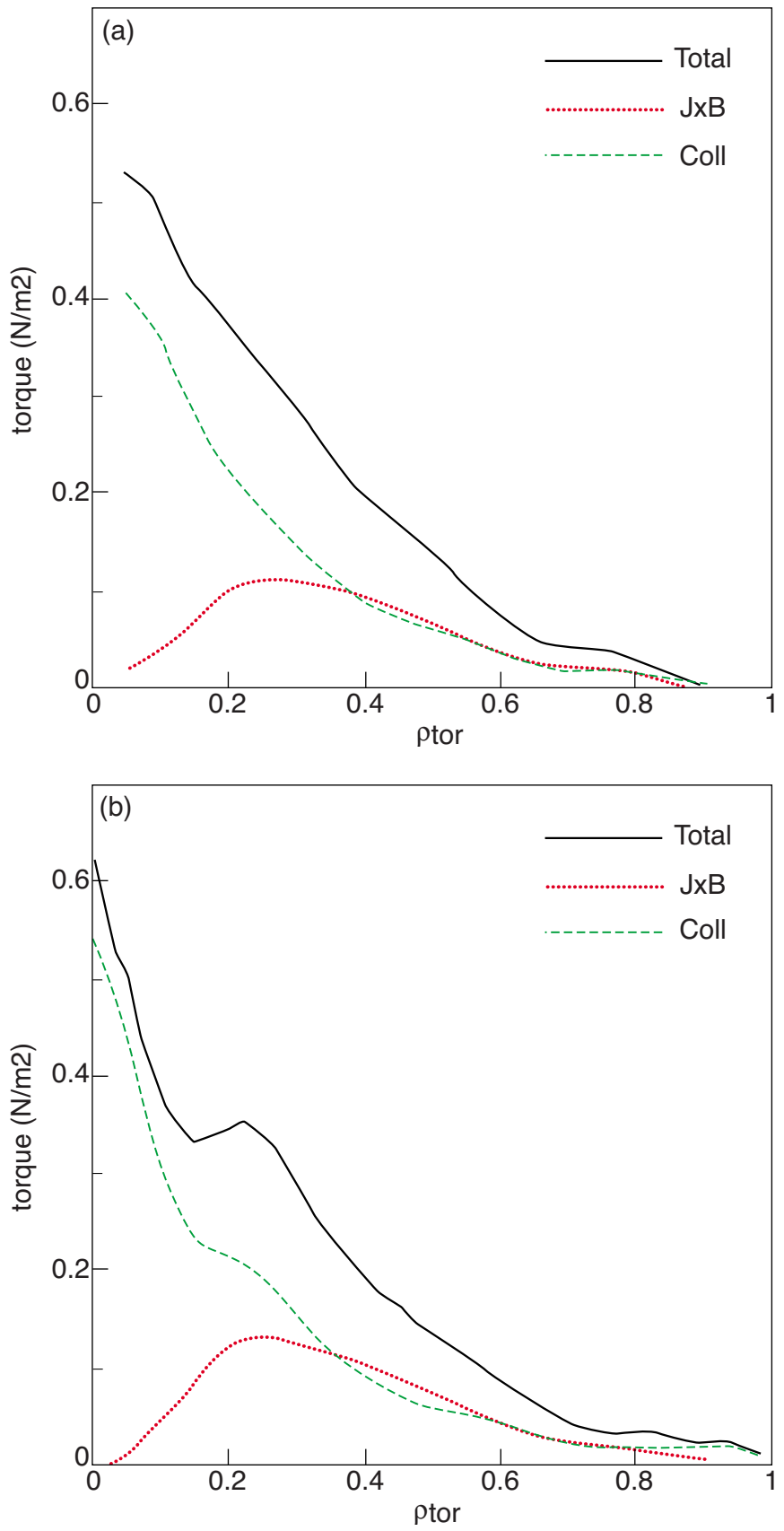

FIG. 11. (Color online) Radial profiles of steady-state torque density in shot 73701 (noncompensated), distinguishing collisional and $\boldsymbol{J} \times \boldsymbol{B}$ components, calculated by (a) TRANSP and (b) ASCOT.

$Z_{\text {eff }}$ have much smaller impact. This is due to the fact that in this discharge the dominant torque is the $\boldsymbol{J} \times \boldsymbol{B}$, which is mainly affected by $n_{\mathrm{e}}$ through a change in ionization profile, while $T_{\mathrm{e}}$ and $Z_{\text {eff }}$ affect the slowing down time of the ions, i.e., only the collisional part of the torque. Particular care has then to be paid in assessing the best $n_{\mathrm{e}}$ profile. For the LIDAR measurements used in this paper the uncertainty on $n_{\mathrm{e}}$ is $\pm 10 \%$ and this has been further improved following the recent introduction of $n_{\mathrm{e}}$ measurements using high resolution Thomson scattering $\left(\Delta n_{\mathrm{e}} \sim \pm 5 \%\right)$. Therefore, we do not expect uncertainties larger than $\pm 4 \%-8 \%$ in torque profiles associated to uncertainties in plasma parameters. Taking into account the uncertainties on $T_{\mathrm{i}}$, rotation, $n_{\mathrm{e}}$, torque, and ion heat fluxes discussed above, we can estimate that $\chi_{\phi}$ and $\chi_{\mathrm{i}}$ are separately determined with $\sim \pm 18 \%$ accuracy and their ratio with $\sim \pm 25 \%$ accuracy.

\section{TRANSPORT ANALYSIS OF TIME-DEPENDENT TOROIDAL ROTATION USING JETTO}

\section{A. The noncompensated modulation case}

\section{Empirical transport model with diffusivity constant in time}

The power and torque sources calculated by TRANSP and described in Sec. III have been used in the transport analysis of toroidal rotation using the $1.5 \mathrm{D}$ code JETTO. ${ }^{36}$ The transport equation for $\omega_{\phi}$ is solved while $q$-profile, $T_{\mathrm{i}}$, $T_{\mathrm{e}}$, and $n_{\mathrm{e}}$ are frozen to their experimental values. The boundary conditions for steady-state $\omega_{\phi}$, amplitudes $A\left(\omega_{\phi}\right)$ and phases $\varphi\left(\omega_{\phi}\right)$ of the modulated $\omega_{\phi}$ are chosen to fit the experimental data at $\rho=0.8$ as the edge plasma transport is beyond the scope of interest in this study. The transport simulations for shot 66128 are carried out over the nine modulation cycles shown in Fig. 1.

The transport model for toroidal momentum is an empirical one, constant in time, featuring a diagonal momentum diffusivity given by $\chi_{\phi}=P_{\mathrm{r}} \cdot \chi_{\mathrm{i}}^{\text {eff }}$ (where $P_{\mathrm{r}}$ is the Prandtl number and $\chi_{\mathrm{i}}^{\text {eff }}$ is the effective ion heat diffusivity, which is a good estimator of the diagonal $\chi_{\mathrm{i}}$, because there is no experimental evidence of an ion heat pinch in JET from existing $T_{\mathrm{i}}$ modulation experiments ${ }^{37,38}$ ) and a pinch velocity $v_{\text {pinch }}$. The values of $P_{\mathrm{r}}$ and $v_{\text {pinch }}$ are chosen in such a way that they fit the available data with sufficient accuracy according to visual inspection (in principle their profiles are completely free with no outside constraints).

The simulations are run with three logical steps: (1) run a fully interpretative simulation to calculate $\chi_{\mathrm{i}}^{\text {eff; }}$ (2) run predictive simulations for $\omega_{\phi}$ varying the $P_{\mathrm{r}}$ value and profile until a reasonable reproduction of the experimental phase profile of the modulated $\omega_{\phi}$ is obtained, as the phase is rather insensitive to $v_{\text {pinch }}$; and (3) vary $v_{\text {pinch }}$ value and profile to reproduce also the amplitude of the modulated $\omega_{\phi}$ and its steady-state profile. Step 1 is straightforward as $\chi_{i}^{\text {eff }}$ can be calculated from the measured $T_{\mathrm{i}}$ data and calculated power deposition profiles. $\chi_{i}$ eff is shown in Fig. 16. Step 2 leads to a rather precise identification of the acceptable range of $P_{\mathrm{r}}$ values, since $P_{\mathrm{r}}$ is the only unknown (the sources are taken from the NUBEAM calculations). This resolves the indeterminacy associated with the analysis of only the steady-state profile, as the latter can be reproduced by an unlimited number of possible combinations for $\chi_{\phi}$ and $v_{\text {pinch }}$ yielding the same $\chi_{\phi}{ }^{\text {eff }}$. Once $P_{\mathrm{r}}$ is identified, step 3 allows us to identify also the convective component that enables us to reproduce the steady-state $\omega_{\phi}$ and amplitude with the chosen $P_{\mathrm{r}}$. As an initial choice, $P_{\mathrm{r}}$ was taken uniform along radius, and as a refinement, $P_{\mathrm{r}}$ has been chosen to have a radial profile, as suggested from the gyrokinetic simulations described in Sec. $\mathrm{V}$, but most of all because it provides a better fit to most of the shots analyzed.

In Fig. 17 we show the simulations of the modulated data of shot 66128 corresponding to the two most obvious options for $\chi_{\phi}$ (i.e., $P_{\mathrm{r}}$ ) and $v_{\text {pinch }}$ : in Fig. 17(a) we fix 


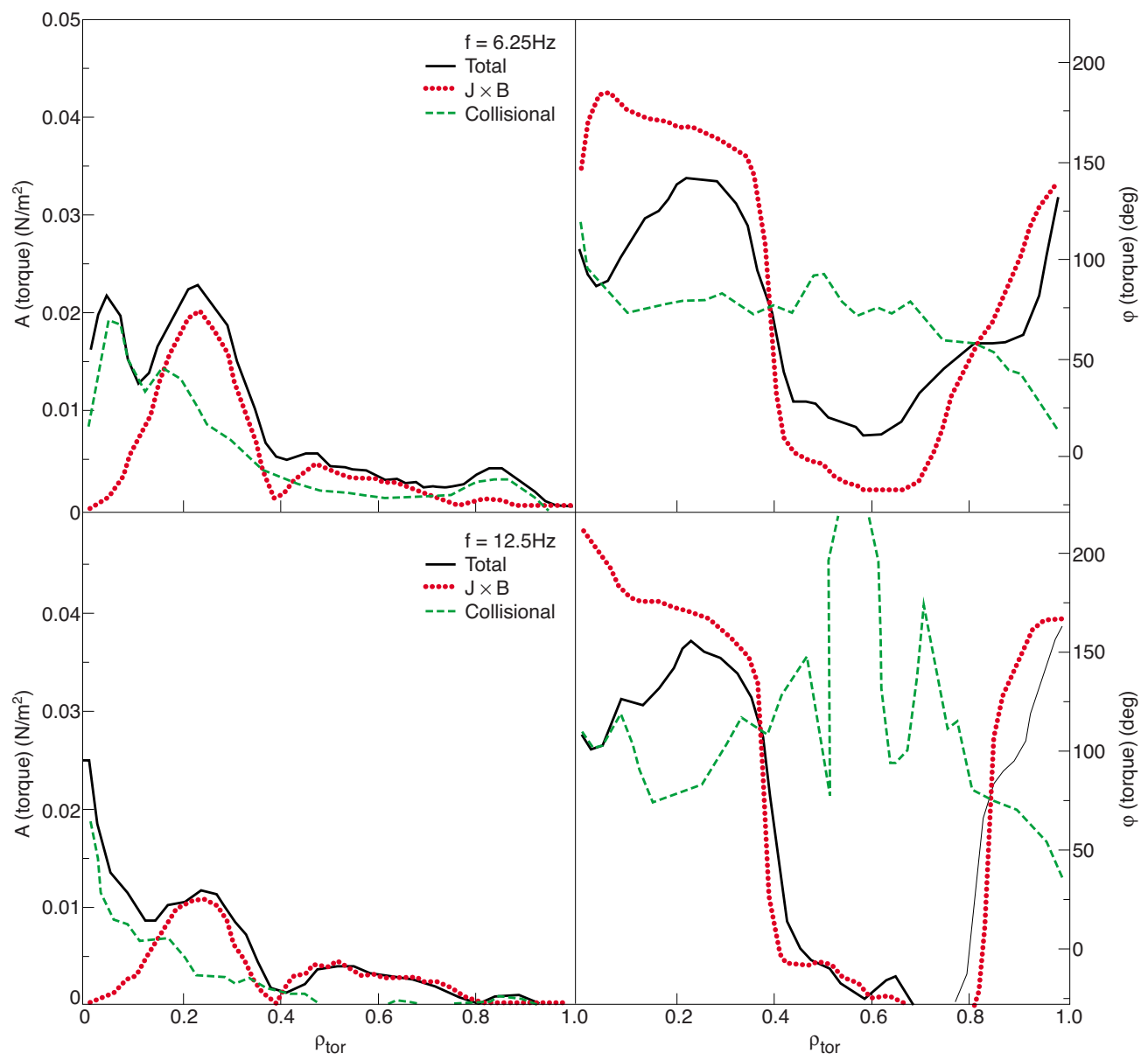

FIG. 12. (Color online) Radial profiles of $A$ and $\varphi$ of TRANSP calculated torque density at 6.25 and $12.5 \mathrm{~Hz}$ in shot 73700 (compensated), distinguishing collisional and $\boldsymbol{J} \times \boldsymbol{B}$ components.

$P_{\mathrm{r}}=0.5$ to yield $\chi_{\phi} \approx \chi_{\phi}{ }^{\text {eff }}$ and $v_{\text {pinch }}=0$ while in Fig. 17(c) we follow the above described best-fit procedure, which leads to $P_{\mathrm{r}} \sim 2$ as the best value to fit the phase profiles, and to the $v_{\text {pinch }}$ profile shown in Fig. 16, with values up to 20 $\mathrm{m} / \mathrm{s}$, to fit amplitudes as well as steady-state. It can be seen in Fig. 17(b) that both simulations predict the steady-state $\omega_{\phi}$ within $10 \%$ accuracy in the region of interest, i.e., 0.2 $<\rho_{\text {tor }}<0.8$. Inside $\rho_{\text {tor }}<0.2$, neoclassical transport starts to dominate ion heat transport, and the predictions are worse as the use of the ITG based $P_{\mathrm{r}}$ for calculating $\omega_{\phi}$ is not appropriate. In Figs. 17(a) and 17(c) one can see that the two options differ in reproducing the $A\left(\omega_{\phi}\right)$ and $\varphi\left(\omega_{\phi}\right)$ profiles. The case with $P_{\mathrm{r}}=0.5$ and $v_{\text {pinch }}=0$ clearly disagrees with the experiment. The simulated phase is too large inside and this is an indication of too low $\chi_{\phi}$, i.e., too low $P_{\mathrm{r}}$ used in the simulation. On the other hand, the simulated amplitude is too low toward the plasma center, which could only be cured by lowering $\chi_{\phi}$ further. This shows that a model with $v_{\text {pinch }}=0$ is not compatible with the experimental data. In the second case, the agreement between the simulated and experimental amplitudes and phases improves dramatically. Using $P_{\mathrm{r}}=2$ the $\chi_{\phi}$ is now large enough to yield perfect match of the phase profile, and the pinch is required to provide the observed amplitude peaking. This is a direct indication of the existence of a convective term in the toroidal momentum transport. The same simulation using, instead of uniform $P_{\mathrm{r}}$, a radially varying $P_{\mathrm{r}}$ profile, as shown in Fig. 16, and with the same $v_{\text {pinch }}$ gives in this case as good agreement with experimental $A$ and $\varphi$ as the $P_{\mathrm{r}}=2$ simulation [Fig. 17(d)], but a better steady-state rotation profile. A sensitivity analysis using the experimental uncertainties on $A$ and $\varphi$ and on plasma parameters and torque source shows that the range of $P_{\mathrm{r}}$ and $v_{\text {pinch }}$ values compatible with such uncertainties is in the range of $20 \%-30 \%$, outside which the simulated phase, amplitude, and steady-state deviate unacceptably from the experimental values.

Another example is the simulation of discharge 73701 (two harmonics available) shown in Fig. 18. This has been simulated using the same procedure and the profiles of $\chi_{\phi}$ and $v_{\text {pinch }}$ derived from the best-fit procedure are shown in Fig. 19. Again, a high $P_{\mathrm{r}}$ number and a significant pinch are required to fit the experimental data.

\section{Effect of fluctuating $\chi_{\mathrm{i}}$ due to $T_{\mathrm{i}}$ oscillations}

A significant complicating factor in the noncompensated experiment is the $T_{\mathrm{i}}$ modulation induced by the NBI power modulation. In fact, being the ITG the supposed drive of toroidal momentum transport, a time varying $T_{\mathrm{i}}$ and/or $R / L_{\mathrm{Ti}}$ implies a time varying ITG driven transport, both for ion heat and momentum. The induced oscillation in $\chi_{\phi}$ produces a component in the rotation modulation, adding an extra con- 


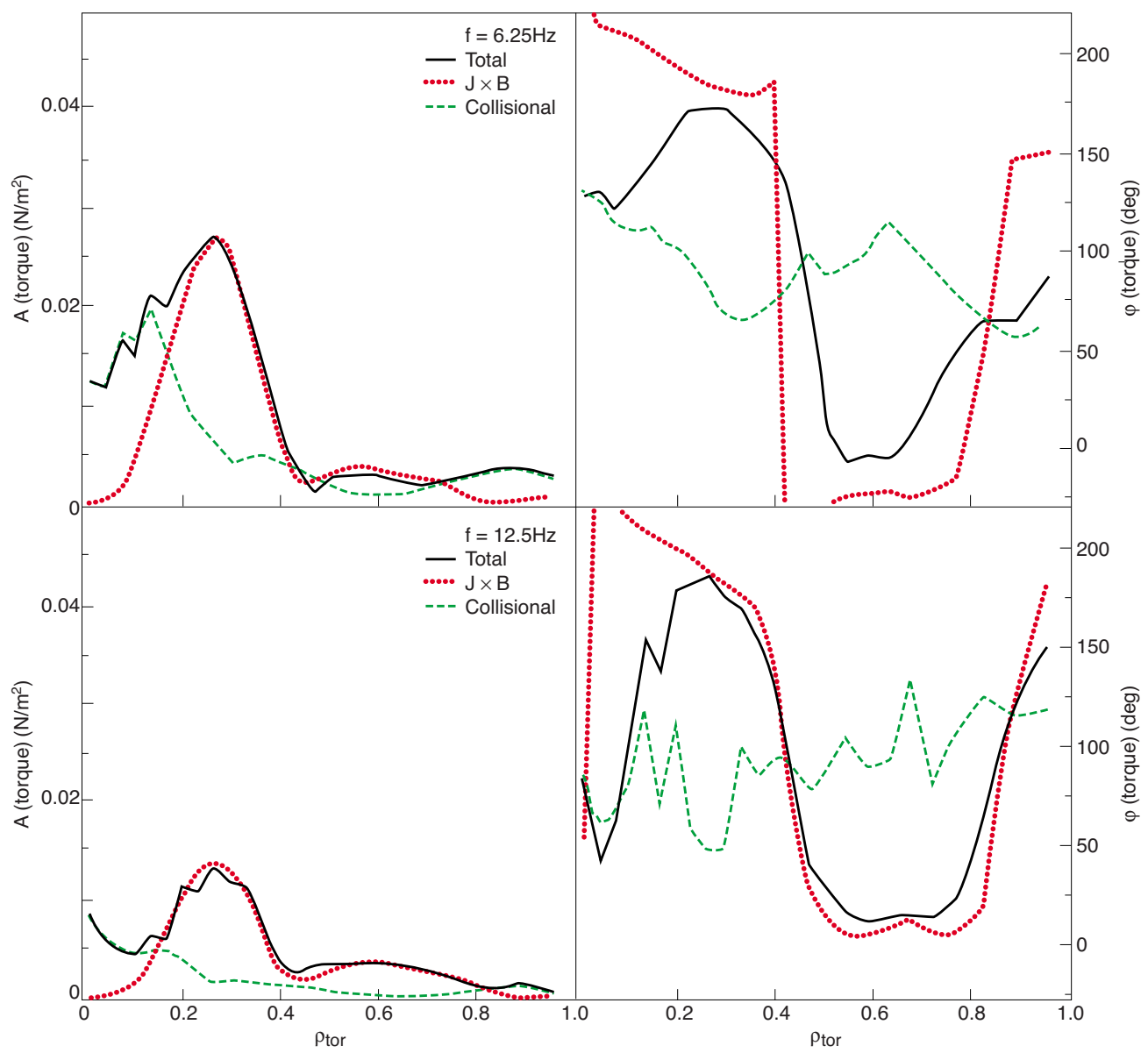

FIG. 13. (Color online) Radial profiles of $A$ and $\varphi$ of ASCOT calculated torque at 6.25 and $12.5 \mathrm{~Hz}$ in shot 73700 (compensated), distinguishing $\boldsymbol{J} \times \boldsymbol{B}$ and collisional beam components.

tribution to the $A\left(\omega_{\phi}\right)$ and $\varphi\left(\omega_{\phi}\right)$ profiles, which, if not properly taken into account, may lead to an erroneous evaluation of the $P_{\mathrm{r}}$ and $v_{\text {pinch }}$ values. Therefore it is important to assess quantitatively the relevance of this mechanism on the results of Sec. IV A 1.

As seen in Figs. 5(b) and 5(c) for shot 66128, the ion and electron temperatures are modulated with peak amplitudes around $70 \mathrm{eV}$, i.e., a perturbation of $\sim 1 \%$, while the modulation amplitude in $\omega_{\phi}$ is around $4 \%$. To estimate the impact of such $T_{\mathrm{i}}$ modulation on the determined $P_{\mathrm{r}}$ and $v_{\text {pinch }}$, a time-dependent $\chi_{\mathrm{i}}$ has been calculated for shot 66128 using the critical gradient model (CGM) model based on the critical gradient length concept. ${ }^{39}$ This model has no implications on momentum transport and here it has been used only to evaluate the expected modulation in $\chi_{\mathrm{i}}$ (and through $P_{\mathrm{r}}$ in $\chi_{\phi}$ ) because it is a model commonly used in JET to investigate ion heat transport from $T_{\mathrm{i}}$ modulation experiments. ${ }^{37,38}$ Typical values for ion threshold and stiffness found in JET in shots similar to the ones discussed here have been used to model the modulated $T_{\mathrm{i}}$ and the associated time variation of $\chi_{\mathrm{i}}$ and $\chi_{\phi}$. The reason why we did not use directly the timedependent experimental $\chi_{i}^{\text {eff }}$ instead of the one modeled by CGM is the very high level of noise that is fed into to the experimental $\chi_{\mathrm{i}}{ }^{\text {eff }}$ by the experimental determination of the $T_{\mathrm{i}}$ gradient. The same noise problems hold for the determination of a time-dependent $\chi_{\Phi}$ directly from the momentum flux and rotation gradient, with in addition the complication of having a non-negligible and unknown convective part. Figure 20 shows the time traces of $\chi_{\mathrm{i}}$ at different radii, Fig. 21(a) the simulated $T_{\mathrm{i}}$ modulation, and Figs. 21(b) and 21 (c) the $\omega_{\phi}$ modulation, with the same two assumptions for $P_{\mathrm{r}}$ and $v_{\text {pinch }}$ as in Figs. 17(b) and 17(d). One can see that the reproduction of $T_{\mathrm{i}}$ modulation in Fig. 21(a) is satisfactory, given the large uncertainties associated with the $T_{\mathrm{i}}$ modulation data after subtraction of the oscillating displacement effect, which for $T_{\mathrm{i}}$ is significant, as was shown in Fig. 5(b). This allows us to assume that the level of oscillation of $\chi_{i}$ in Fig. 20 is representative of the amount of effect to be expected, even if we cannot have a precise determination of threshold and stiffness level for this shot. One can also see that the simulations of $\omega_{\phi}$ with oscillating $\chi_{\mathrm{i}}$ (and consequently $\chi_{\phi}$ ) in Figs. 21(b) and 21(c) do not differ significantly from their counterparts with constant $\chi_{i}$ in Figs. $17(b)-17(d)$. One can conclude that, owing to the small amplitude of the $T_{\mathrm{i}}$ modulation and of the induced $\chi_{\mathrm{i}}$ and $\chi_{\phi}$ modulation, the effect on the values determined for $P_{\mathrm{r}}$ and $v_{\text {pinch }}$ is insignificant. The same conclusion is actually derived from the first principles simulations using the quasilinear fluid Weiland model ${ }^{40}$ discussed in Sec. VI. 


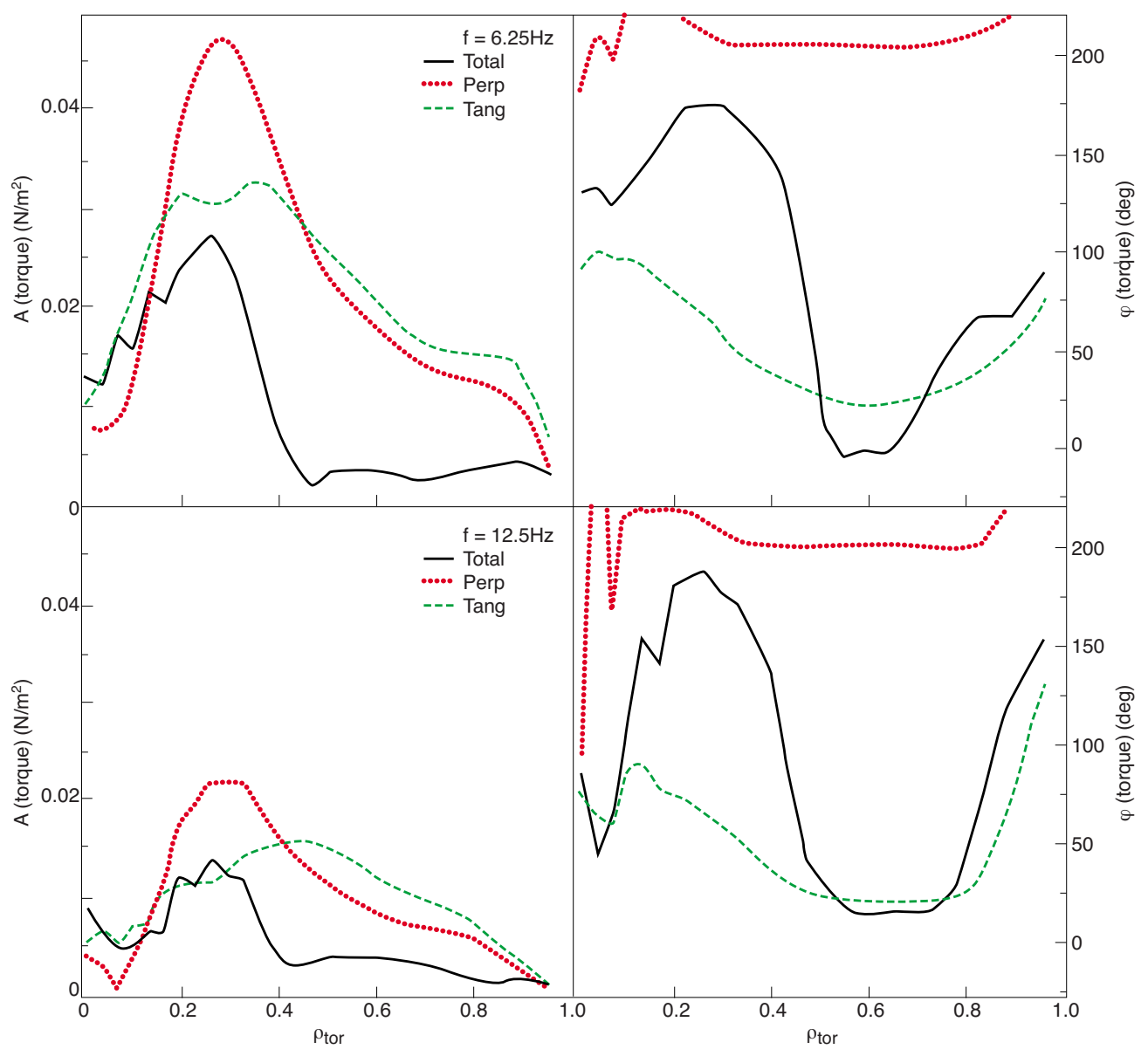

FIG. 14. (Color online) Radial profiles of $A$ and $\varphi$ of ASCOT calculated torque at 6.25 and $12.5 \mathrm{~Hz}$ in shot 73700 (compensated), distinguishing tangential and perpendicular beam components.

\section{B. The compensated modulation case}

The same procedure described in Sec. IV A 1 has been applied to the compensated modulation case, shot 73700 , using the TRANSP time-dependent torque described in Sec. III. In this case the logic behind the best-fit is however completely different, because it is no longer true that phases are uniquely determined by diffusivities. In fact, best-fitting the data has turned out very cumbersome, because the phase profile is the result of two propagating waves (normal and tangential beams) of modulated rotation, in antiphase with each other, so that the amplitude of each component plays a dominant role in determining the resultant phase, and therefore momentum pinch enters in the phase dynamics as well. The core being dominated by the normal beams, while the outer region by the tangential, the rapidity of the increase by $180^{\circ}$ of the phase between the two components, depends crucially on the Prandtl number: low $P_{\mathrm{r}}$ number yields a very fast transition, while high $P_{\mathrm{r}}$ number a smooth one, with the pinch value determining the precise phase profile. In addition, a low $P_{\mathrm{r}}$ number would yield two very well defined peaks of amplitudes, while a high $P_{\mathrm{r}}$ number would tend to smooth them down. It is clear from the data shown in Fig. 7 that experimentally the transition is very smooth, and there is no pronounced peak of the tangential component, which are nontrivial confirmations of the presence of a high $P_{\mathrm{r}}$ number (implying then a pinch in order to match the steady-state). The best simulation achieved for the compensated shot 73700 is compared against the data in Fig. 22 and shows a fair agreement in profile and magnitude for modulated and steady-state data. The $P_{\mathrm{r}}$ numbers and pinch profiles used in the simulation are shown in Fig. 23. We conclude that the compensated modulation supports the previous conclusions about the existence of a pinch. In general, however, this procedure has turned out too cumbersome to be used for a systematic study of several shots such as when performing parametric scans. In addition, we note that the $\mathrm{S} / \mathrm{N}$ level in such compensated modulation is much lower than in the noncompensated case, as amplitudes are reduced due to compensation. In particular outside $\rho=0.5$, which is the region where the pinch is expected to be largest, the signal is very near to noise level, so the sensitivity of this technique to the pinch value is reduced. Since on the other hand the problem of modulated power is not found to seriously affect the data of the noncompensated case, as discussed in Sec. IV A, the compensated technique has been abandoned at JET and the noncompensated one has been used for further studies of parametric dependencies of momentum transport, which will be the object of a future paper. 


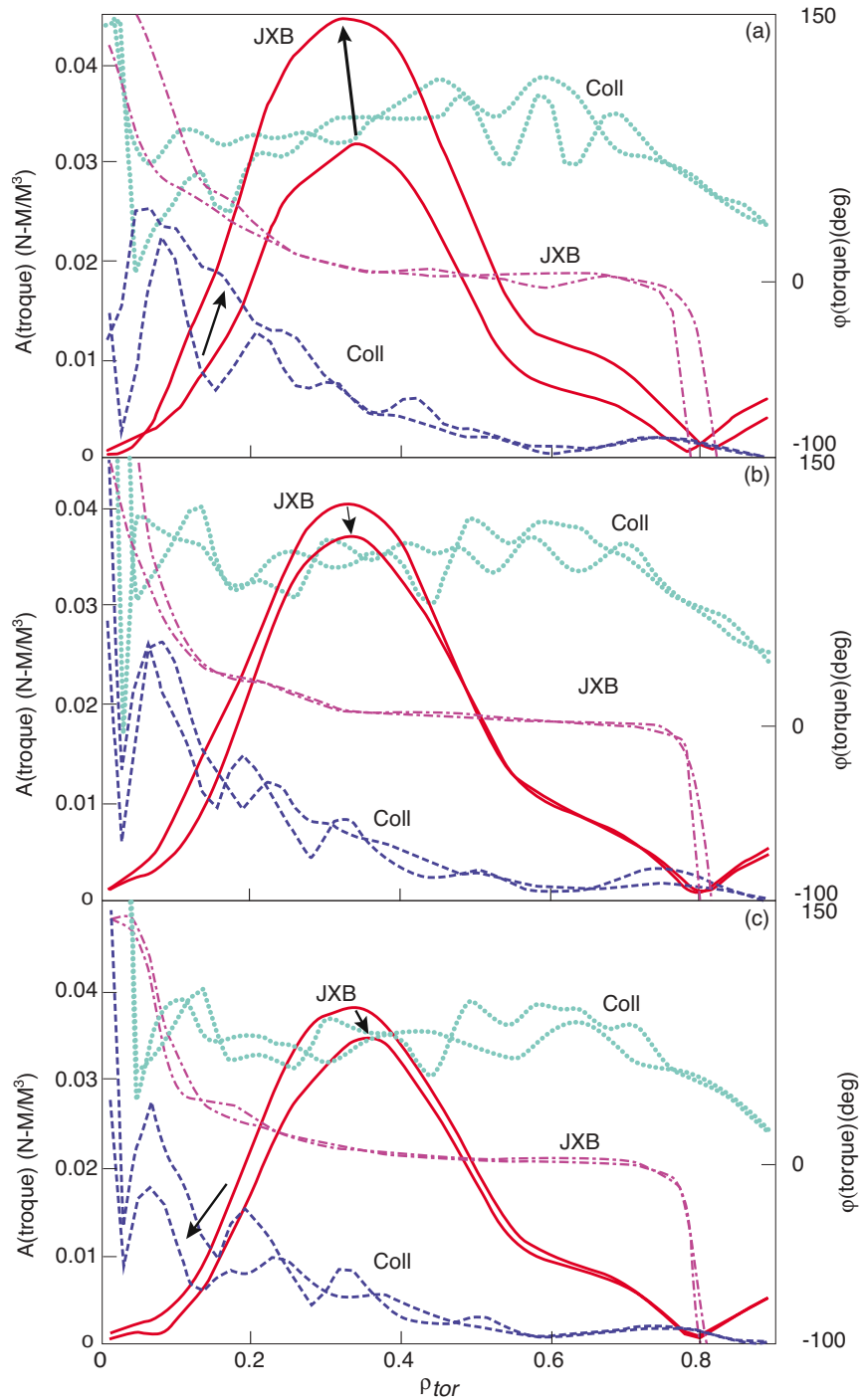

FIG. 15. (Color online) Radial profiles of $A$ and $\varphi$ of TRANSP calculated torque at $8.33 \mathrm{~Hz}$ in shot 73701 with (a) $n_{\mathrm{e}}$ varying between 0.8 and 1.2 of experimental value; (b) $T_{\mathrm{e}}$ varying between 0.8 and 1.2 of experimental value; and (c) $Z_{\text {eff }}$ varying between 1.9 and 1 . Red full lines are $A$ of $\boldsymbol{J} \times \boldsymbol{B}$ torque, blue dashed lines are $A$ of collisional torque, violet dasheddotted lines are $\varphi$ of $\boldsymbol{J} \times \boldsymbol{B}$ torque, and green dotted lines are $\varphi$ of collisional torque. The arrows indicate the relevant changes due to parameter variation.

\section{THEORETICAL PREDICTIONS OF TOROIDAL MOMENTUM TRANSPORT FROM LINEAR GYROKINETIC SIMULATIONS}

Effective transport of momentum needs, like all turbulent transport, a phase shift between the turbulent velocity fluctuations and the momentum fluctuations. Such a phase shift is established by a finite average rotation but also generally through symmetry breaking, in which case residual stresses or asymmetry momentum fluxes arise. ${ }^{7,9}$ For negligible residual stress terms, the resulting equation for the transport of momentum can be directly written as the sum of a diffusive and pinch contribution in the local limit. Offdiagonal terms, i.e., a momentum flux directly driven by the density and temperature gradients, have been investigated in global simulations in Ref. 41 and were found to be one order smaller in normalized Larmor radius and therefore negligible for JET parameters.

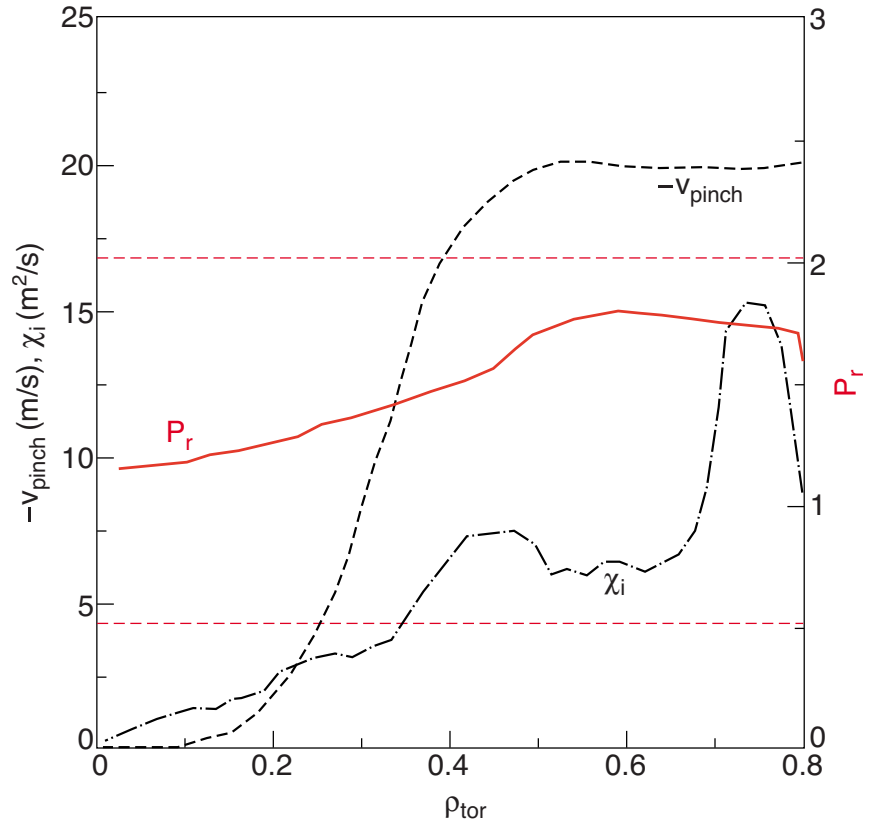

FIG. 16. (Color online) Radial profiles of $\chi_{\mathrm{i}}$, ${ }^{\text {eff }}, v_{\text {pinch }}$, and $P_{\mathrm{r}}$ used in the simulations of shot 66128 discussed in the text.

Gyrokinetic studies of the diffusion coefficient $\chi_{\phi}$ are presented in Refs. 8 and 11, whereas the pinch term $v_{\text {pinch }}$ was derived in Ref. 9 using the comoving reference frame (for more details on the derivation see Ref. 42). In the comoving frame the toroidal rotation enters the equations only through the Coriolis drift velocity. Consequently, any toroidal momentum pinch is directly related to the Coriolis drift. Since this drift, unlike the drifts due to the magnetic field inhomogeneity, is linear in the parallel velocity, it generates a coupling between density/temperature perturbations and the perturbations in the parallel velocity. Over this coupling the temperature perturbations of the ITG generate parallel velocity perturbations that are then transported by the $\boldsymbol{E} \times \boldsymbol{B}$ velocity leading to a finite radial flux of parallel momentum. Furthermore, the drift in the electrostatic potential perturbations accelerates the particles and also leads to the generation of parallel velocity fluctuations and consequently to a momentum flux. Although no complete expression for the flux has been derived for the laboratory frame, the latter effect is related to the turbulent equipartition (TEP) (Ref. 43) theory and has recently been worked out in Ref. 10. Obviously, the calculated radial fluxes should be frame independent and it can be shown that the TEP contribution is incorporated in the description that uses the Coriolis drift (see Ref. 44).

The gyrokinetic flux tube code GKW (Ref. 45) is used here to calculate the values of the momentum transport coefficients under experimental conditions. All parameters are taken directly from the experiment, but the following approximations have been made. (a) The geometry is approximated using the $s-\alpha$ model, with the circular radius chosen such that the number of trapped particles is roughly equal to the experimental case. This approximation is motivated by having found negligible impact of full with respect to circular geometry on the ITG simulations made for very similar discharges within the ion heat transport studies described in 

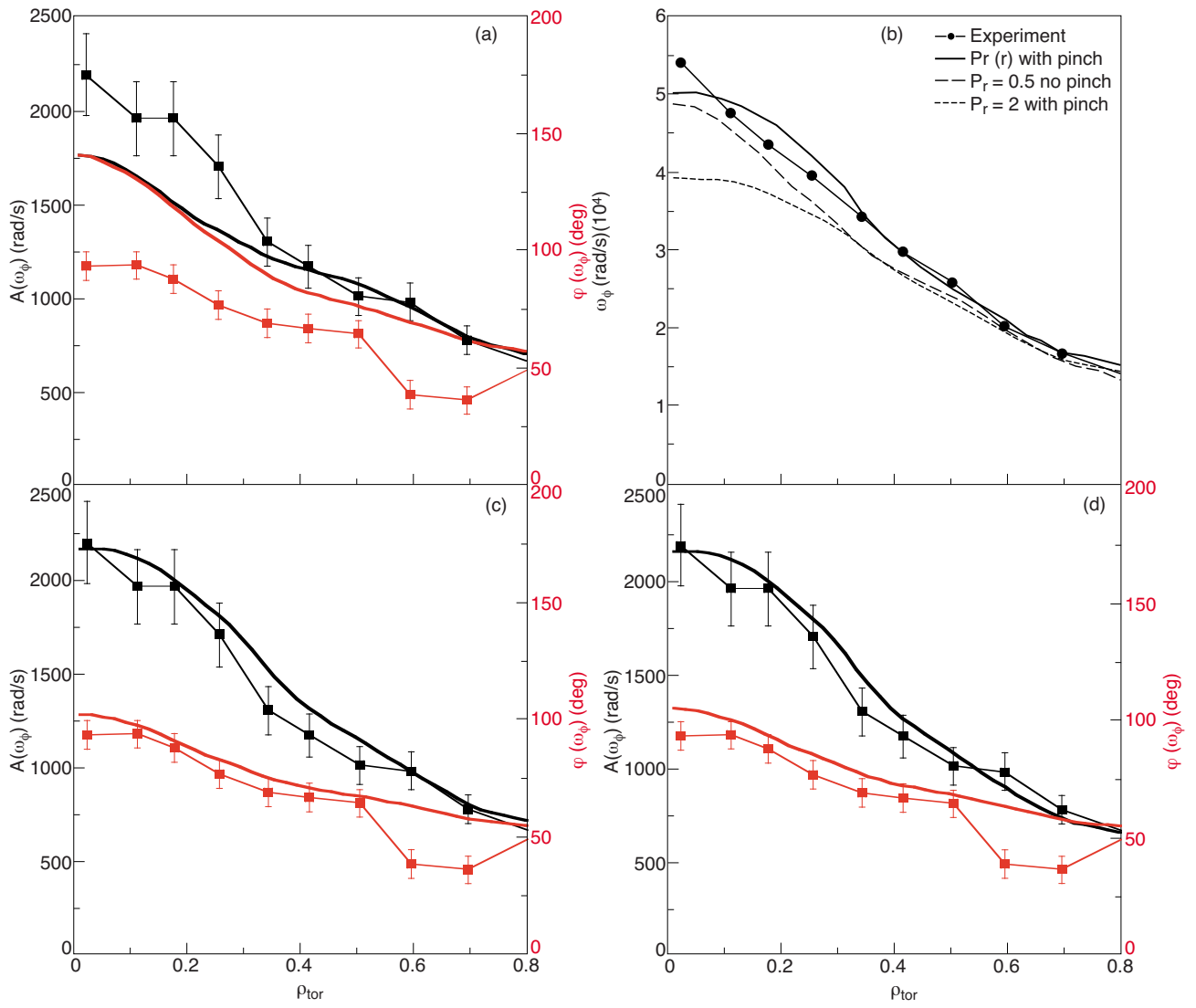

FIG. 17. (Color online) Radial profiles of experimental (dots) and simulated (lines) $A$ (black squares) and $\varphi$ (red circles) of $\omega_{\phi}$ in shot 66128 using (a) $P_{\mathrm{r}}=0.5$ and $v_{\text {pinch }}=0 \mathrm{~m} / \mathrm{s}$; (c) $P_{\mathrm{r}}=2$ and $v_{\text {pinch }}$ as shown in Fig. 16; (d) a $P_{\mathrm{r}}$ profile increasing with radius (Fig. 16) and $v_{\text {pinch }}$ as shown in Fig. 16. In (b) the radial profiles of experimental and simulated angular rotation using the three different transport options are shown.

Ref. 37. (b) A pure deuterium plasma is considered and Coulomb collisions are neglected. The latter approximation is motivated by the small influence of the collisions. ${ }^{46}$ The simulations presented here have been performed for the most unstable mode $k_{\theta} \rho_{\mathrm{s}}=0.3$.

The $P_{\mathrm{r}}$ number $\left(\chi_{\phi} / \chi_{\mathrm{i}}\right)$ and the pinch number $\left(R v_{\text {pinch }} / \chi_{\phi}\right)$ profiles calculated by GKW are compared with the experimental ones in Figs. 24 and 25 for the JET pulses 66128 and 73701, respectively. The determination of both Prandtl number and pinch requires two simulations per radial position: one with zero rotation $\left(v_{\phi}=0\right)$ and one with zero gradient ( $\operatorname{grad} \nu_{\phi}=0$ ). In the latter case the diagonal contribution is zero, and hence the momentum flux, which is obtained from the code directly, gives the pinch contribution $\left(v_{\text {pinch }}=\Gamma_{\phi} / n v_{\phi}\right)$, whereas in the former case the pinch contribution is zero and the momentum flux gives information on the diffusion coefficient $\left(\chi_{\phi}=-\Gamma_{\phi} / n \operatorname{grad} v_{\phi}\right)$. Of course, this procedure assumes that the fluxes are linear in both $v_{\phi}$ and grad $\nu_{\phi}$, an assumption that is well satisfied as shown in Ref. 9.

One can see that for shot 73701 (low density) the predicted and experimental Prandtl and pinch numbers are in good agreement with experiment, although only a statistical study outside the scope of this work would be able to assess whether such agreement is real or fortuitous. However in shot 66128 (higher density) experimentally the reduction in $\chi_{\mathrm{i}}$ due to increased density is larger than the one in $\chi_{\phi}$, thus

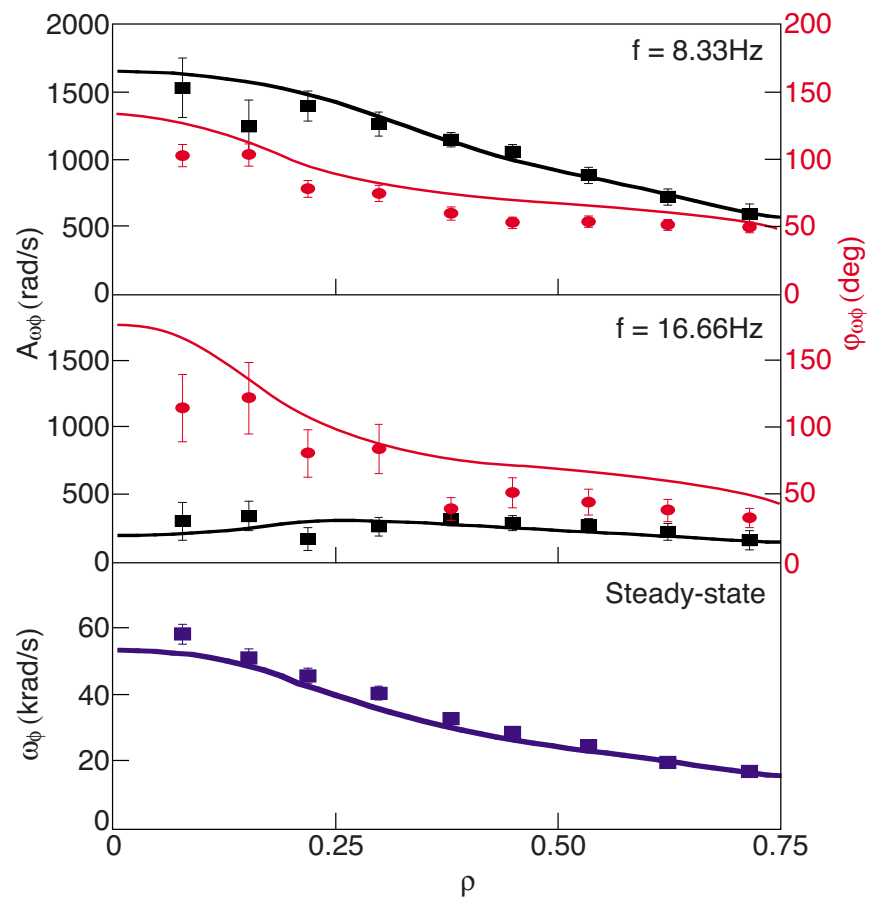

FIG. 18. (Color online) Radial profiles of experimental (dots) and simulated (lines) $A$ (black squares) and $\varphi$ (red circles) of $\omega_{\phi}$ at two harmonics of the modulation frequency and steady-state $\omega_{\phi}$ in shot 73701. The simulation derives (by best-fitting the data) the $P_{\mathrm{r}}$ and $v_{\text {pinch }}$ profiles, as shown in Fig. 19. 


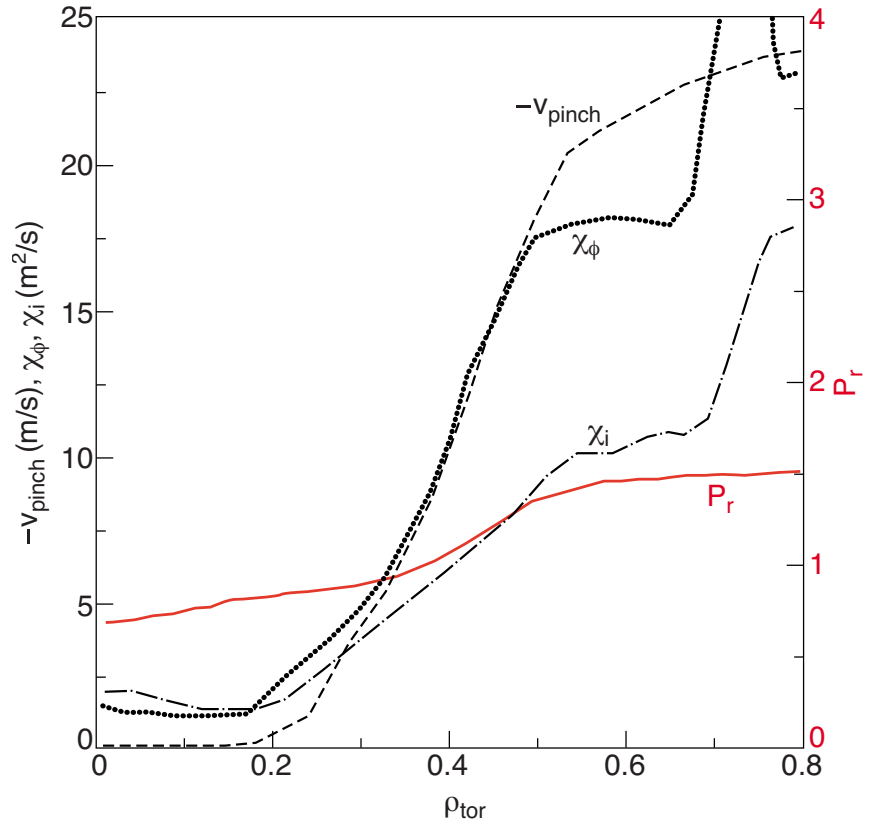

FIG. 19. (Color online) Radial profiles of $\chi_{\phi}, \chi_{\mathrm{i}},-v_{\text {pinch }}$, and $P_{\mathrm{r}}$ from the empirical simulation of shot 73701 .

giving origin to higher experimental $P_{\mathrm{r}}$ and pinch numbers, while the theory prediction remains basically unchanged. Therefore a discrepancy by a factor of 1.5-2 is present in this latter shot between GKW estimates and experiment. This seems a trend with density which however will be further investigated with more shots in a future work. On the other hand, the theoretical description of momentum transport is still evolving and at present it may already be quite satisfactory to find an agreement with experiment within a factor of 2. Indeed more than one reason can be put forward. Nonlin-

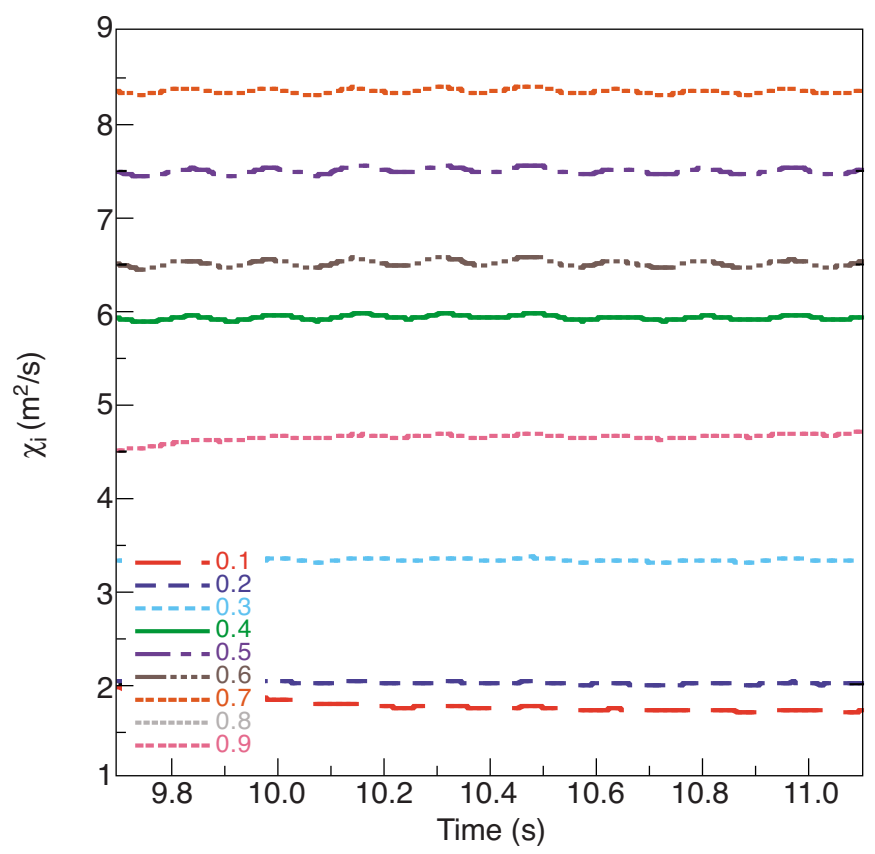

FIG. 20. (Color online) Time traces of $\chi_{\mathrm{i}}$ at different radii as calculated for shot 66128 using the CGM model with typical values of ion threshold and stiffness derived from previous ion heat transport studies.

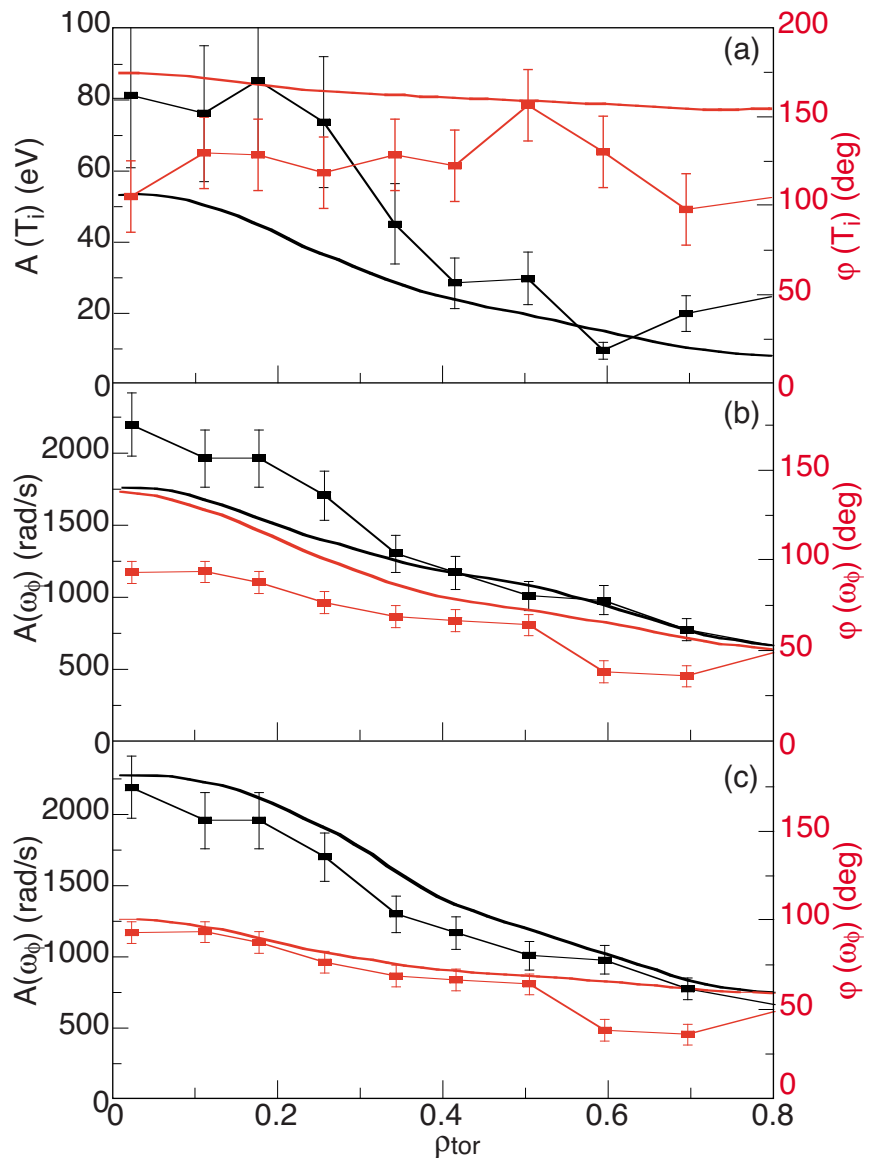

FIG. 21. (Color online) Radial profiles in shot 66128 of experimental (dots) and simulated (lines) $A$ and $\varphi$ of (a) $T_{\mathrm{i}}$ using $\chi_{\mathrm{i}}$ from the CGM model; (b) $\omega_{\phi}$ using $P_{\mathrm{r}}=0.5$ and $v_{\text {pinch }}=0 \mathrm{~m} / \mathrm{s}$ and $\chi_{\mathrm{i}}$ from the CGM model; and (c) $\omega_{\phi}$ using the $P_{\mathrm{r}}$ profile increasing with radius (Fig. 16) and $v_{\text {pinch }}$ as shown in Fig. 16 and $\chi_{\mathrm{i}}$ from the CGM model.

ear effects, for instance, might lead to different transport coefficients. Furthermore, several effects such as the $\boldsymbol{E} \times \boldsymbol{B}$ shearing, ${ }^{47}$ the current symmetry breaking, ${ }^{48}$ and the residual stress ${ }^{49}$ are not kept in the current description. It is clear then that further theory development and also more extensive validation against data are required, in view of improving our predictive capabilities. It is also fair, however, to recognize that theory has made substantial steps forward in a field little explored until recently, and for which the exercise of validation against data has just started. The agreement within a factor of 2 found within this first and challenging exercise of validation against perturbative momentum transport experiments is therefore encouraging.

Finally we remark that with linear simulations only normalized quantities can be compared with experiment, but not absolute values of transport coefficients. This will have to wait for nonlinear gyrokinetic simulations of momentum transport. This observation justifies the attempt made in Sec. VI to compare with experiment the predictions of the quasilinear fluid Weiland model, which can be used for a full transport simulation of the time evolution of rotation, thereby allowing a complete comparison with experiment. 


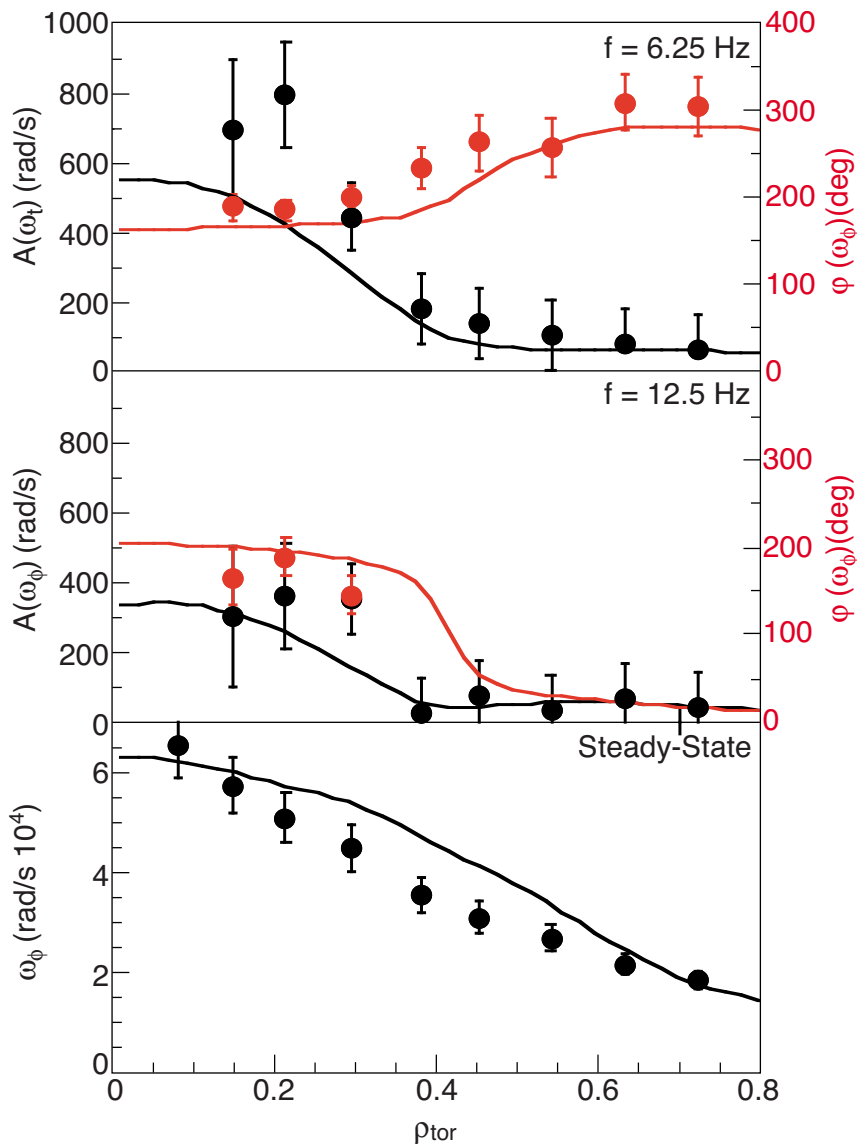

FIG. 22. (Color online) Radial profiles of experimental (dots) and simulated (lines) $A$ (black squares) and $\varphi$ (red circles) of $\omega_{\phi}$ at two harmonics of the modulation frequency and steady-state $\omega_{\phi}$ in shot 73700 (compensated case). The simulation derives (by best-fitting the data) the $P_{\mathrm{r}}$ and $v_{\text {pinch }}$ profiles shown in Fig. 23.

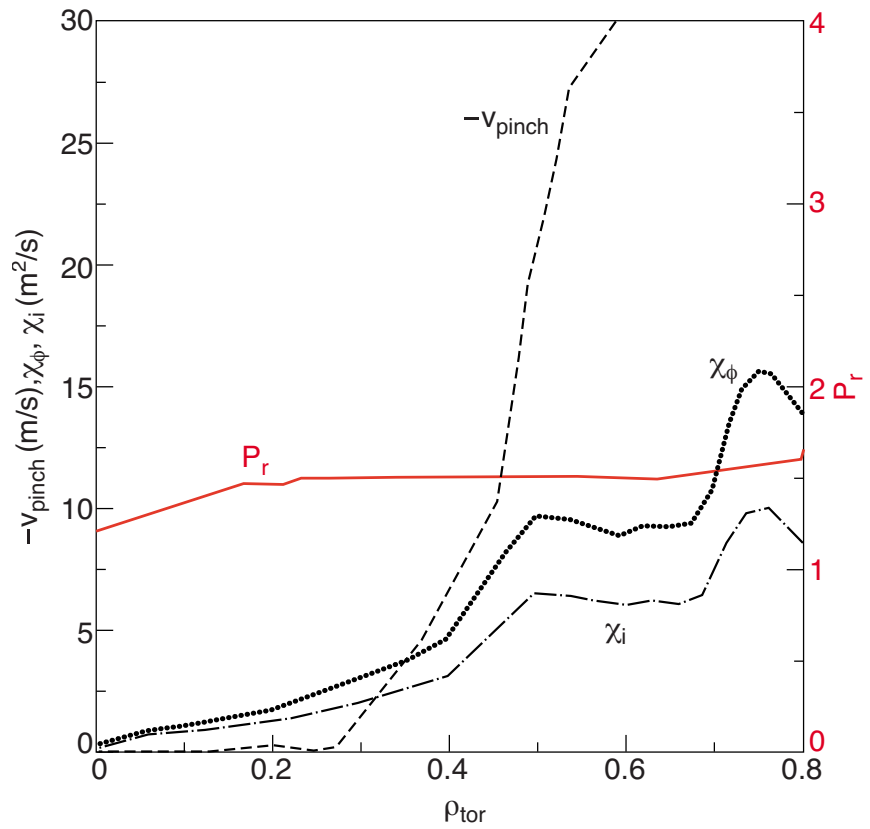

FIG. 23. (Color online) Radial profiles of $\chi_{\phi}, \chi_{\mathrm{i}},-v_{\text {pinch }}$, and $P_{\mathrm{r}}$ from the empirical simulation of shot 73700 .

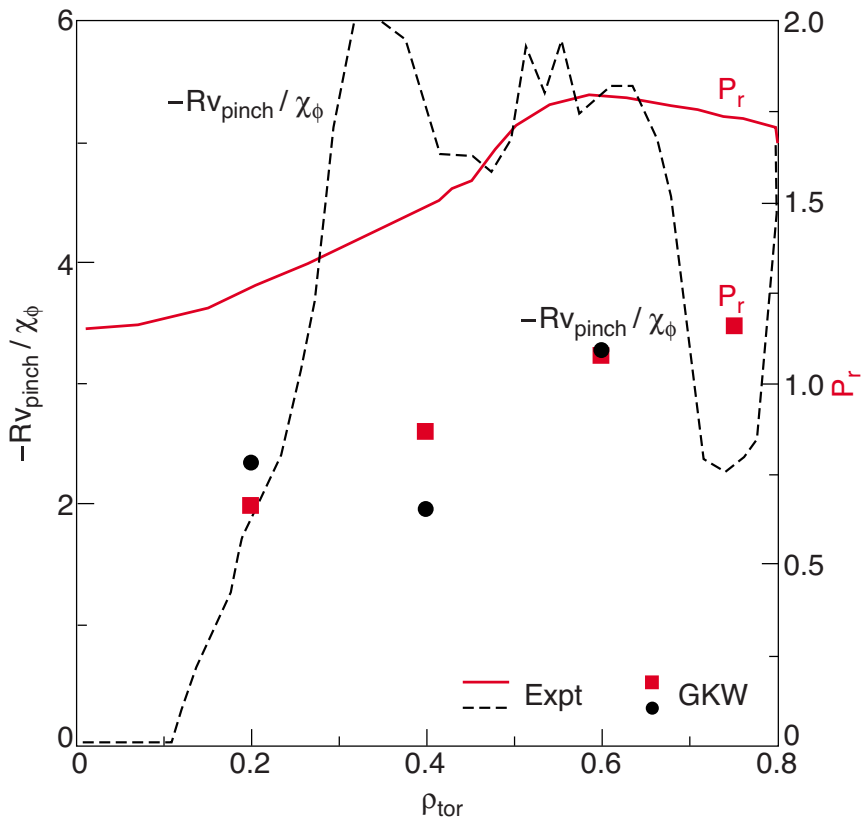

FIG. 24. (Color online) Comparison between experiment and linear gyrokinetic simulations using GKW of the radial profile of $P_{\mathrm{r}}$ and $-R v_{\text {pinch }} / \chi_{\phi}$ for shot 66128 .

\section{TIME-DEPENDENT FLUID MODELING}

This section describes first attempts to do timedependent simulations of the JET NBI modulation experiments using the quasilinear toroidal drift wave fluid model described in Ref. 40, which due to the inclusion of a space dependent nonlinear frequency shift (giving zonal flows) is no longer quasilinear, in which a new treatment of momentum transport has been recently implemented, ${ }^{12}$ making it unique in the class of quasilinear momentum transport mod-

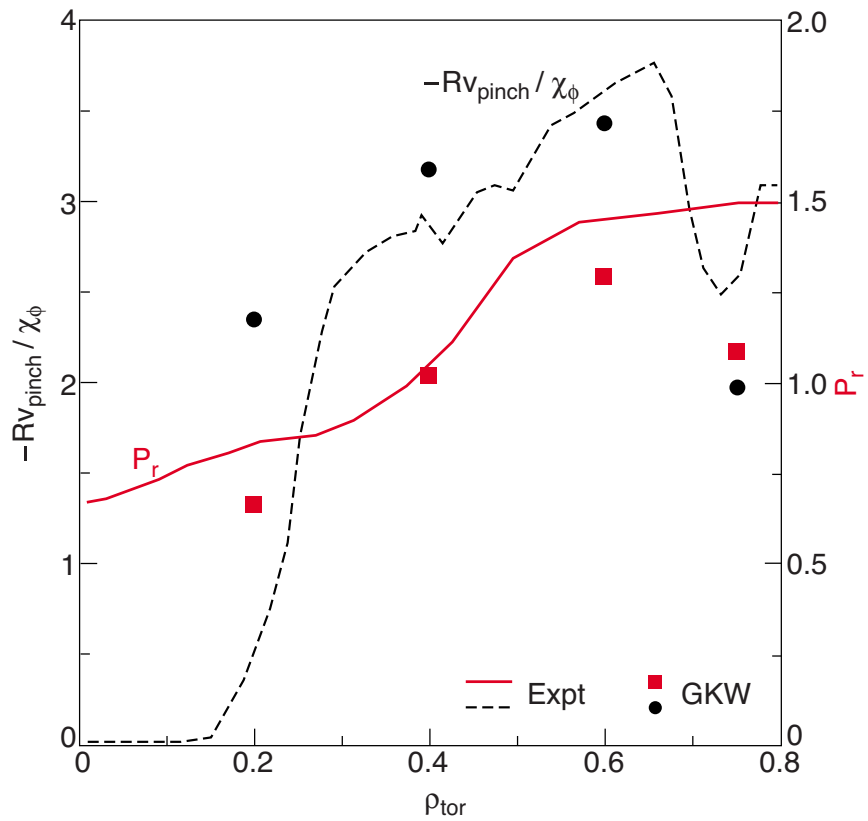

FIG. 25. (Color online) Comparison between experiment and linear gyrokinetic simulations using GKW of the radial profile of $P_{\mathrm{r}}$ and $-R v_{\text {pinch }} / \chi_{\phi}$ for shot 73701 . 
els available for time-dependent simulations of rotation. New aspects are the inclusion of stress tensor effects for the toroidal momentum. ${ }^{9-12}$ As it turns out, toroidal effects from the stress tensor ${ }^{9}$ are important for the parallel ion motion which we here use as an approximation for the toroidal motion. The toroidal curvature effects enter both for the diagonal and convective parts of the momentum transport:

$$
\begin{aligned}
& m\left[\frac{\partial}{\partial t}+\left(\mathbf{v}_{E}+2 \mathbf{v}_{D}\right) \cdot \nabla\right] v_{\|} \\
& \quad=e\left[E_{\|}+(\vec{v} x \vec{B})_{\|}\right]-\frac{1}{n} \mathbf{e}_{\|} \cdot \nabla P-\frac{1}{n} \nabla \cdot \Pi_{\text {off }} .
\end{aligned}
$$

Here the diagonal contributions from the stress tensor were included explicitly but the off-diagonal contributions are still kept in the remaining part $\Pi_{\text {off }}$ of the stress tensor. The diagonal element of the toroidal momentum transport becomes $^{9,12}$

$$
\chi_{\phi}=\frac{\gamma^{3} / k^{2}}{\left(\omega_{r}-2 \omega_{D i}\right)^{2}+\gamma^{2}} .
$$

The convective fluxes are here taken from the kinetic formulation in Ref. 10. The same fluxes have, however, recently been derived from a fluid formulation in Ref. 12. They are divided into TEP (Refs. 10 and 44)

$$
\Gamma_{\mathrm{TEP}}=\operatorname{Re}\left\{\frac{\left\langle k_{\|}\right\rangle c_{s}^{2}-V_{\| 0} \varpi_{D e}}{\omega-2 \varpi_{D i}} \hat{\phi} \mathbf{v}_{E r}{ }^{*}\right\}
$$

and thermodiffusion

$$
\Gamma_{\text {term }}=\operatorname{Re}\left\{\frac{\left\langle k_{\|}\right\rangle c_{s}^{2}-V_{\| 0} \varpi_{D e}}{\omega-2 \varpi_{D i}} \frac{\delta p}{\tau P} \mathbf{v}_{E r}^{*}\right\} .
$$

The magnetic drift frequency has here been averaged over the mode profile as indicated by the bar in $\varpi_{D i}$. Moreover $\left\langle k_{\|}\right\rangle$has been averaged over the radial mode profile. It is nonzero due to symmetry breaking effects of flowshear and magnetic curvature. The expression is quite complicated and given in Ref. 10. The curvature parts of Eqs. (4) and (5) usually dominate except close to marginal stability.

Since the diagonal element for the ion thermal conductivity is obtained from Eq. (3) by replacing 2 in the denominator by $5 / 3$, we realize that the Prandtl number of the diagonal components has to be very close to 1 . Of the new convective parts, the thermodiffusion is usually the dominant part and is also usually directed inward. As compared to a model without the toroidal stress tensor effects, both diagonal and convective fluxes are considerably larger in the present model. Although the steady-state sometimes does not change very much with the new model, the transient transport does.

This model has been used for 1.5D transport simulations of the JET discharges 66128 and 73701 (noncompensated NBI modulation cases) described previously. Timedependent simulations were carried out by predicting $\omega_{\phi}, T_{\mathrm{i}}$, $T_{\mathrm{e}}$ and keeping $n_{\mathrm{e}}$ fixed to the experimental time-averaged steady-state values. Figure 26 shows the rotation steady-state (a) and modulation $A$ and $\varphi$ (b) for shot 66128, comparing the experiment with the simulation. Figure 26(c) shows for

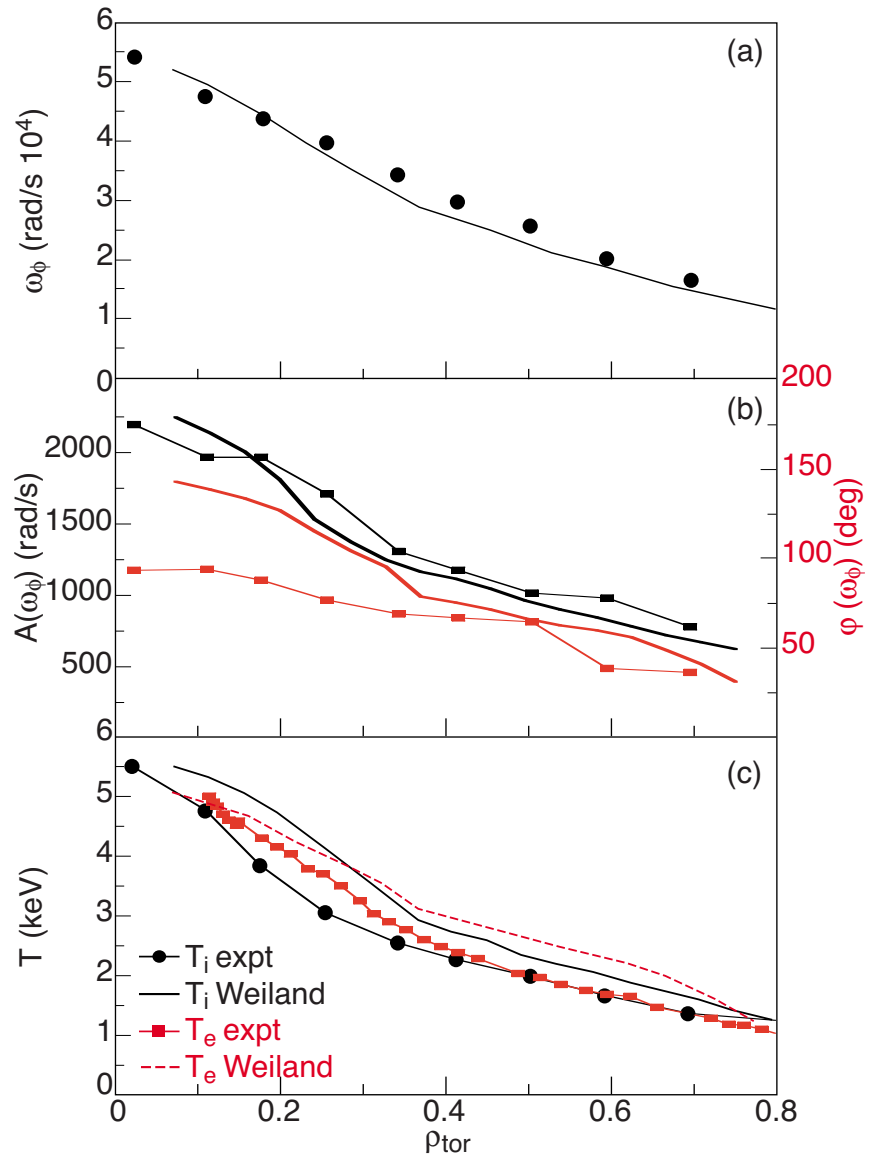

FIG. 26. (Color online) Radial profiles in shot 66128 of experimental (dots with line) and simulated (lines only) (a) time-averaged steady-state $\omega_{\phi}$; (b) $A$ and $\varphi$ of $\omega_{\phi}$; and (c) time-averaged steady-state $T_{\mathrm{i}}$ and $T_{\mathrm{e}}$. The simulations are carried out using the Weiland model with $T_{\mathrm{i}}, T_{\mathrm{e}}$, and $\omega_{\phi}$ predicted and $n_{\mathrm{e}}$ fixed to the experimental profile.

the same simulation the profiles of $T_{\mathrm{i}}$ and $T_{\mathrm{e}}$ at a given time. Figure 27 shows the radial profiles of turbulent $\chi_{\mathrm{i}}$ (not including the neoclassical component), diagonal and effective (the simulation in fact foresees the existence of an ion heat pinch), of momentum diffusivity $\chi_{\phi}$, diagonal and effective, and of momentum convective velocity $v_{\text {pinch }}$ predicted by the model. For comparison also the experimental $\chi_{i}^{\text {eff }}$ and $\chi_{\phi}$ diagonal and $v_{\text {pinch }}$ are plotted. We note that in the model the diagonal $\chi_{\mathrm{i}}$ and also $\chi_{\phi}$ [Eq. (3)] depend on the eigenfrequency. Through that they depend also on other gradients and thus their definition as diagonal elements is not really appropriate. This may be particularly important for this model where diagonal and effective ion thermal conductivities differ significantly. In Fig. 28 the $P_{\mathrm{r}}$ and pinch number from the simulation (both defined using the diagonal diffusivities for $\chi_{\phi}$ and $\chi_{\mathrm{i}}$ ) and from experiment (where we use the diagonal $\chi_{\phi}$ but the effective $\chi_{\mathrm{i}}$ since so far we have no experimental evidence of an ion heat pinch) are compared. Figures 29-31 show the same quantities (excluded the $T_{\mathrm{i}}$ and $T_{\mathrm{e}}$ profiles) for shot 73701 . One can see that for both shots the model predicts the existence of a momentum pinch, however, quantitatively in absolute magnitude significantly smaller than found in experiment $\left(v_{\text {pinch }} \sim 3-7 \mathrm{~m} / \mathrm{s}\right.$ in simulations against $v_{\text {pinch }} \sim 10-20 \mathrm{~m} / \mathrm{s}$ in experiment). Also the 


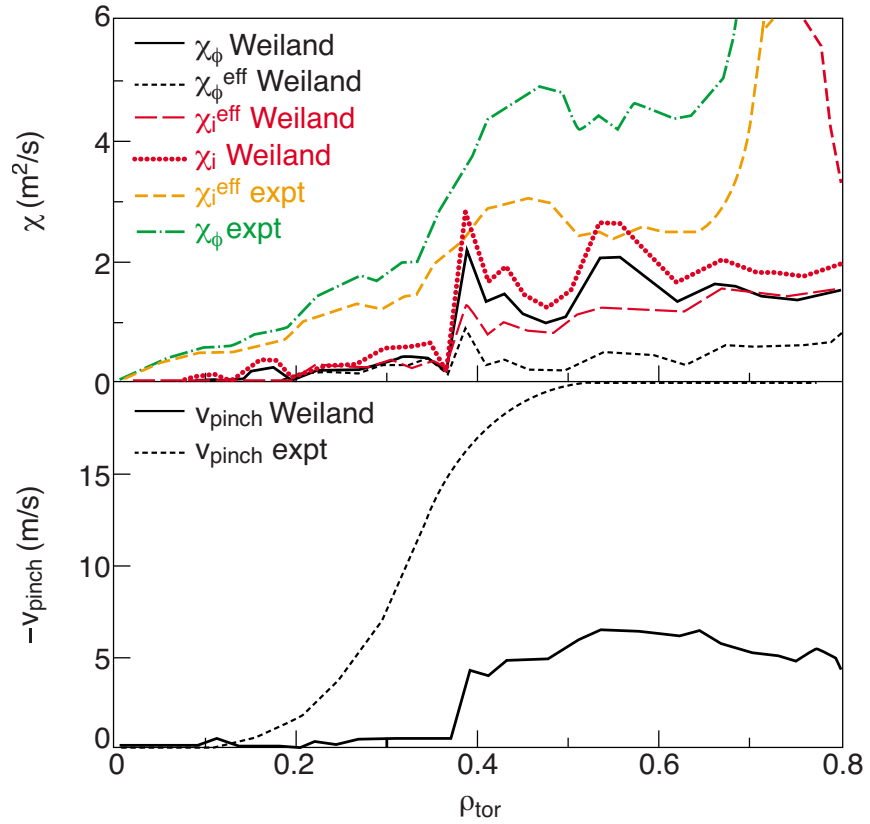

FIG. 27. (Color online) Radial profiles of turbulent $\chi_{\mathrm{i}}$ (diagonal and effective), $\chi_{\varphi}$ (diagonal and effective), and momentum $v_{\text {pinch }}$ for the Weiland model simulation of shot 66128 shown in Fig. 25, compared with profiles of effective $\chi_{\mathrm{i}}$ (turbulent+neoclassical), diagonal $\chi_{\varphi}$, and $v_{\text {pinch }}$ obtained by best-fitting experimental data.

absolute value of the diagonal $\chi_{\phi}$ is significantly smaller in the simulations, which gives origin to steeper simulated phase profiles in Figs. 26 and 29. So in spite of a good agreement with experiment of steady-state rotation profiles and amplitudes, the disagreement on the phases clearly indicates that both momentum diffusivity and pinch are too low. Interestingly, the $P_{\mathrm{r}}$ and pinch number are not far off with respect to experiment, because $\chi_{\mathrm{i}}, \chi_{\phi}$, and $v_{\text {pinch }}$ are all too low, and their ratios remain close to the experimental ones

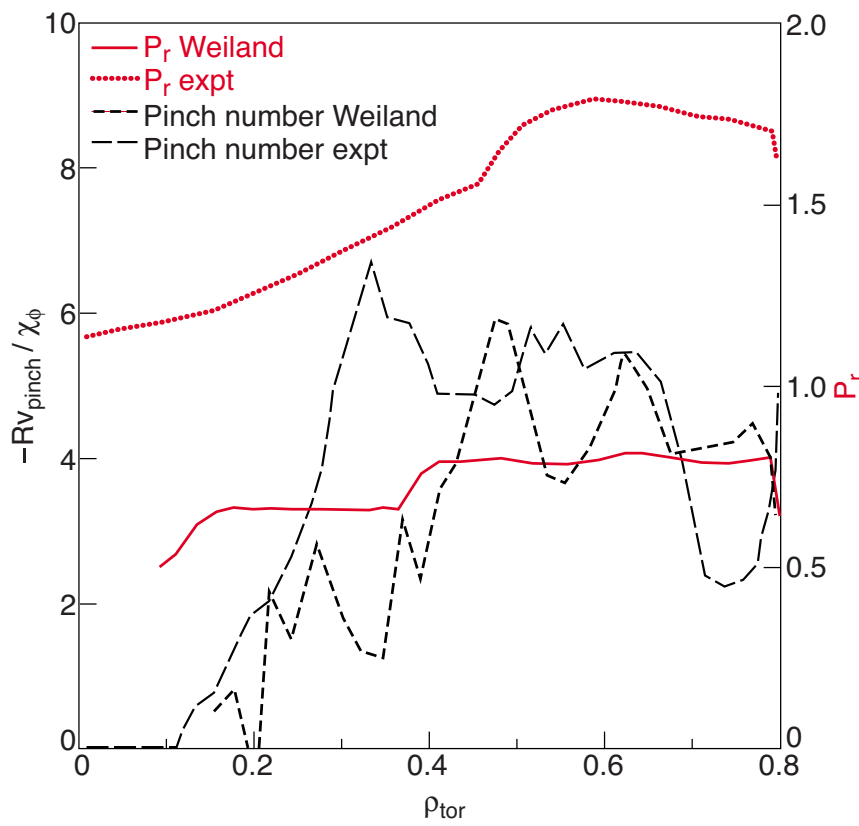

FIG. 28. (Color online) Comparison of radial profiles of Prandtl and pinch numbers from Weiland simulation and experiment for shot 66128 .

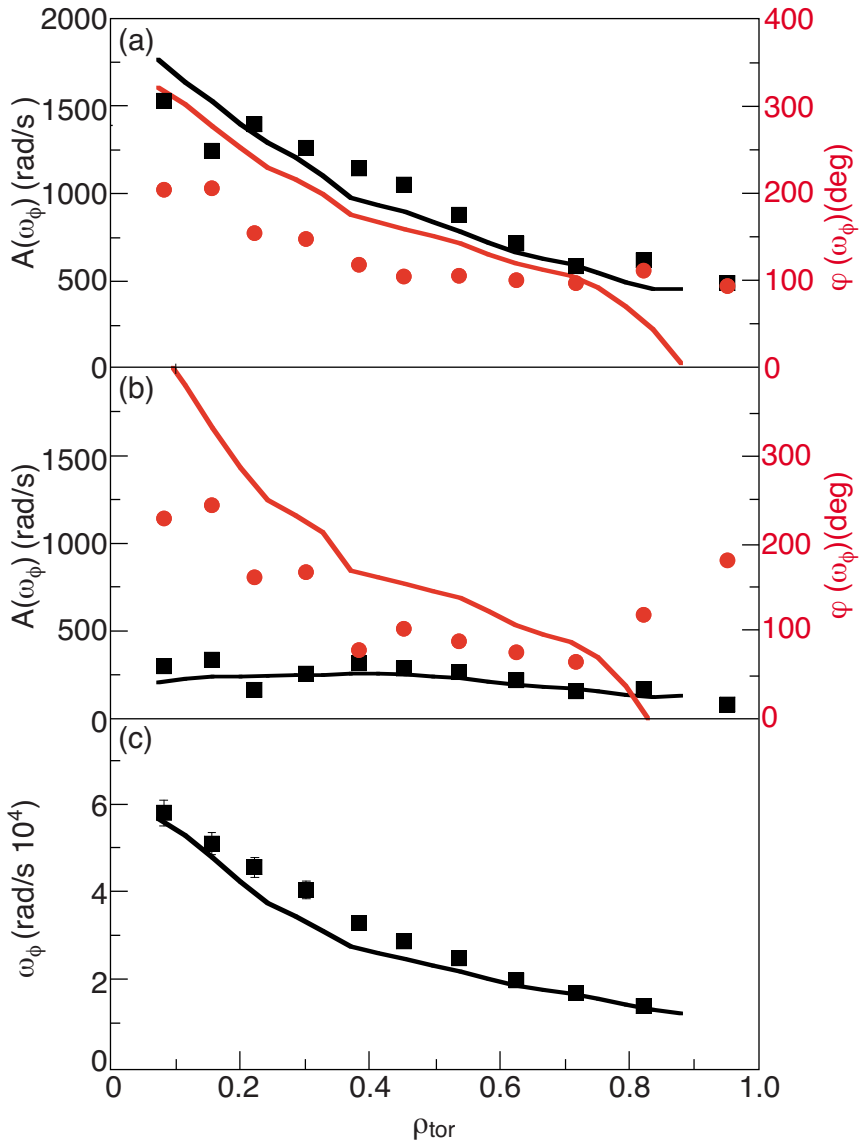

FIG. 29. (Color online) Radial profiles of experimental (dots) and simulated (lines) time-averaged $\omega_{\phi}(\mathrm{c})$ and its $A$ (black squares) and $\varphi$ (red circles) at first (a) and second (b) harmonics in shot 73701 using the Weiland model with $T_{\mathrm{i}}, T_{\mathrm{e}}$, and $\omega_{\phi}$ predicted and $n_{\mathrm{e}}$ fixed to the experimental profile.

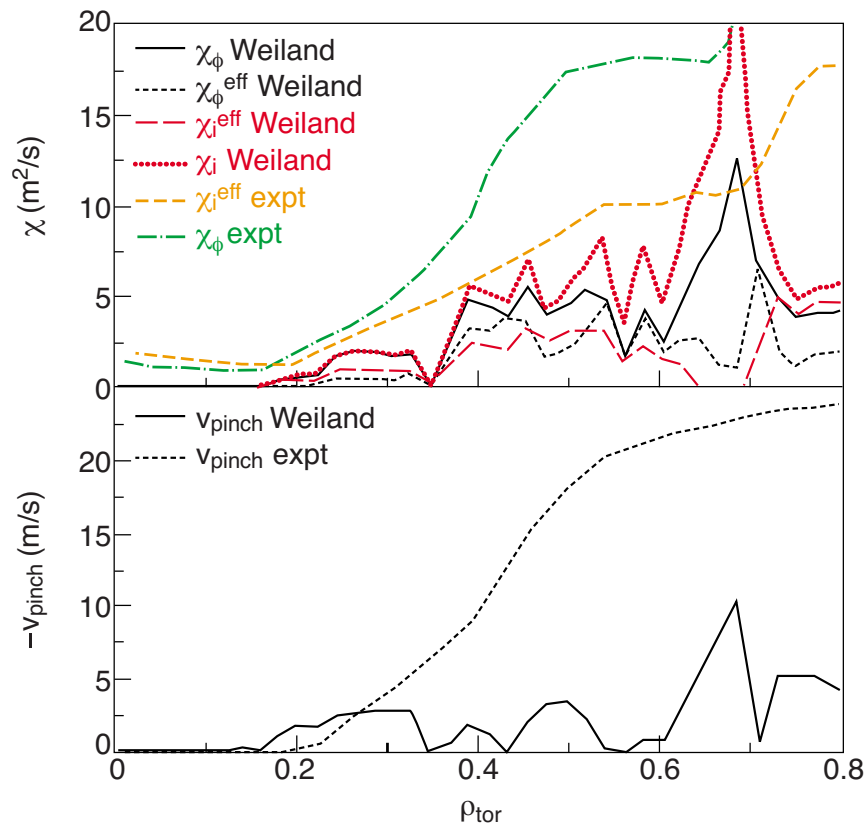

FIG. 30. (Color online) Radial profiles of turbulent $\chi_{\mathrm{i}}$ (diagonal and effective), $\chi_{\varphi}$ (diagonal and effective), and momentum $v_{\text {pinch }}$ for the Weiland model simulation of shot 73701 shown in Fig. 29, compared with profiles of effective $\chi_{\mathrm{i}}$ (turbulent+neoclassical), diagonal $\chi_{\varphi}$, and $v_{\text {pinch }}$ obtained by best-fitting experimental data. 


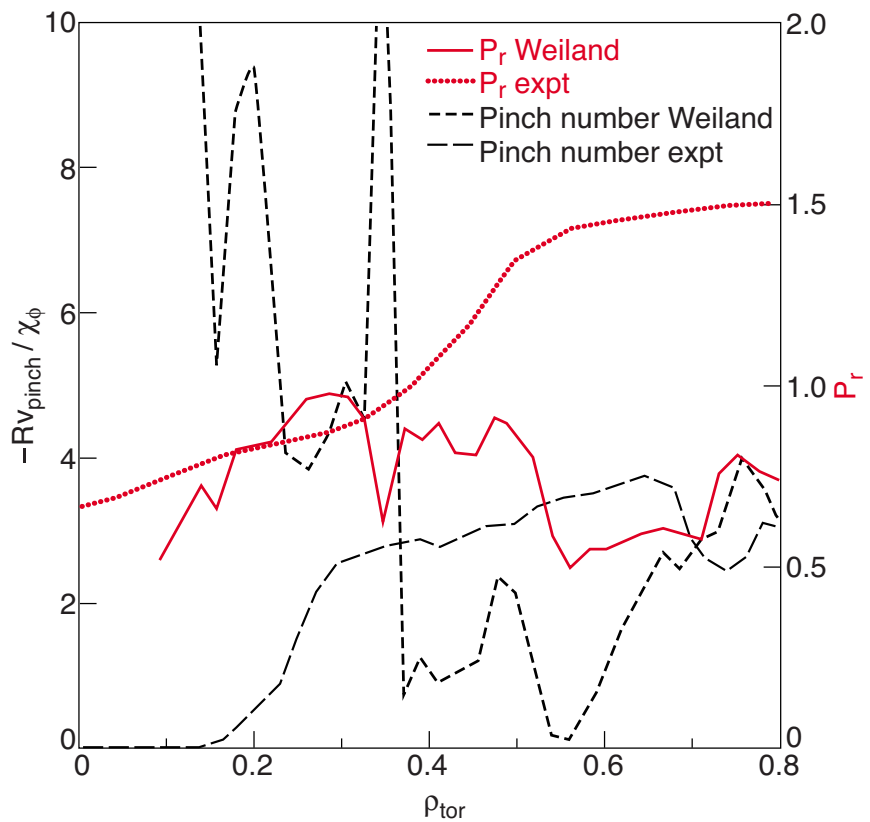

FIG. 31. (Color online) Comparison of radial profiles of Prandtl and pinch numbers from Weiland simulation and experiment for shot 73701 .

(and to the GKW ones). This shows clearly that it is not sufficient to compare only dimensionless ratios to validate a model, but we need also the absolute values of the diffusivities. This is thus a question of overall normalization of the transport, a question which has not been addressed by the GKW results. We remark that also for the Weiland model as for GKW the agreement is better at low density, where the $P_{\mathrm{r}}$ number matches the experimental one at least in the inner part of the plasma (with a different, rather flat profile of $P_{\mathrm{r}}$ number, at variance with GKW), while at high density the experimental $P_{\mathrm{r}}$ number is well above the simulated one.

\section{CONCLUSIONS}

This paper describes in detail the technique set-up at JET to perform perturbative studies of momentum transport using NBI modulation. Experimentally the simplest method of square wave modulation of $\sim 5 \mathrm{MW}$ of $\mathrm{NBI}$ power has turned out the most robust to be used in future studies. It gives a good $\mathrm{S} / \mathrm{N}$ for the rotation modulation, and complicating effects associated to power modulation (such as modulated plasma displacement and modulated ion heat transport) have been shown small and can in any case be accounted for in the analysis. The idea of compensating the power modulation by modulating in antiphase normal and tangential beams yields a much poorer $\mathrm{S} / \mathrm{N}$, requires a rather cumbersome fitting procedure of the data, and was shown to be less sensitive to the pinch value in the region of interest.

The method set-up for extracting the toroidal momentum diffusivity and pinch values is a full time-dependent simulation of a number of NBI modulation cycles using the JETTO code, with empirically adjusted profiles of Prandtl number and pinch in order to fit both the steady-state rotation profiles and amplitudes and phases at two harmonics of the rotation modulation. The key for the uniqueness of the result is that phases are dominantly sensitive to $P_{\mathrm{r}}$ number, while ampli- tudes and steady-state also to the pinch. The main source of uncertainty in the analysis is represented by the need of assuming the torque deposition profile from numerical computations. Therefore a lot of effort has been dedicated to sensitivity studies of the time-dependent torque calculations using TRANSP-NUBEAM Monte Carlo code. A comparison with the time-dependent torque calculated by a totally independent tool, the orbit following Monte Carlo code ASCOT, has given further confidence on the reliability of the calculated torque source. An overall uncertainty of $\sim 20 \%$ on Prandtl number and $\sim 30 \%$ on pinch value has been estimated.

The physics results yielded by the analysis of NBI modulation data have provided valuable insight into momentum transport, allowing to solve the apparent discrepancy between the low effective momentum diffusivity $\left(P_{\mathrm{r}}^{\mathrm{eff}}\right.$ $\sim 0$.4) measured in steady-state experiments, and the ITG based theory prediction of a $P_{\mathrm{r}}$ number close to 1 . In fact, it is clear from the phase response to the modulation that the diagonal momentum diffusivity is fairly large $\left(P_{\mathrm{r}} \sim 1-2\right)$ and that the low effective diffusivity is due to the presence of a significant momentum pinch $\left(v_{\text {pinch }}\right.$ up to $20 \mathrm{~m} / \mathrm{s}$ in the outer part of the plasma). This finding, on one hand, is in very good qualitative agreement with the recent theory developments described in Secs. V and VI; on the other hand it opens interesting perspectives for the possibility of having peaked rotation profiles also in machines with low torque input, allowing to exploit the beneficial effects of flow shear.

For this reason the experimental results for two shots have been compared with linear gyrokinetic predictions of $P_{\mathrm{r}}$ number and pinch number using the code GKW. In addition, the full time evolution of the rotation during several modulation cycles has been computed with the recently updated quasilinear Weiland model for momentum transport and compared with steady-state, amplitude, and phase profiles. The GKW theoretical predictions are in good agreement with data both in value and profile shape for the lower density shot, while the higher density shot has a larger experimental $P_{\mathrm{r}}$ number than predicted by the theory. The reason for the high experimental $P_{\mathrm{r}}$ number is that the increasing density reduces $\chi_{\mathrm{i}}$ more than $\chi_{\varphi}$, in experiment, thereby inducing a significant variation of $P_{\mathrm{r}}$ number. The theory predicted $P_{\mathrm{r}}$ number is instead rather insensitive to variations in main plasma parameters. The reason for such discrepancy remains to be investigated with further experiments at varying plasma parameters such as density, collisionality, and safety factor. Future work will also address the dependence of the momentum pinch on plasma parameters in both experiment and theory. We note also that linear simulations do not yield absolute values of diffusivity and pinch, so it is not possible to compare quantitative predictions of transport with data until nonlinear simulations are available. This is the reason why full simulations of the data using the Weiland model have been attempted. This is presently the only fluid model that has incorporated recent theoretical advances in momentum transport. The main result is that although the dimensionless $P_{\mathrm{r}}$ and pinch number are rather similar to the GKW values and to experiment, the absolute values of $\chi_{\mathrm{i}}, \chi_{\varphi}$, and pinch are all too low compared to experiment, thereby yielding steeper profiles of amplitude and phases. Another observa- 
tion is that the $P_{\mathrm{r}}$ profile is rather flat in the Weiland model, while the experimental and GKW ones are increasing with radius. These modeling results indicate that further work is needed both in terms of theory development and validation against experiment toward the availability of a physics based model for momentum transport to be used for extrapolation to future devices. On the other hand, this first exercise of detailed validation of recent theory developments against challenging data as are those of perturbative experiments is a first encouraging step forward, since it indicates the qualitative agreement of the new theory predictions with experiment as far as the existence of a sizeable momentum pinch is concerned, and also a basic quantitative agreement.

\section{ACKNOWLEDGMENTS}

This work, supported by the European Communities under the contract of Association between EURATOM/ENEACNR, was carried out within the framework of the European Fusion Development Agreement. The views and opinions expressed herein do not necessarily reflect those of the European Commission.

${ }^{1}$ Nucl. Fusion 47(6) (2007).

${ }^{2}$ H. Biglari, P. H. Diamond, and P. Terry, Phys. Fluids B 2, 1 (1990).

${ }^{3}$ R. L. Miller and R. E. Waltz, Phys. Plasmas 1, 2835 (1994).

${ }^{4}$ K. H. Burrell, Phys. Plasmas 4, 1499 (1997).

${ }^{5}$ A. M. Garofalo, M. S. Chu, E. D. Fredrickson, M. Gryaznevich, T. H. Jensen, L. C. Johnson, R. J. La Haye, G. A. Navratil, M. Okabayashi, J. T. Scoville, E. J. Strait, A. D. Turnbull, and DIII-D Team, Nucl. Fusion 41, 1171 (2001).

${ }^{6}$ J. W. Berkery, S. A. Sabbagh, R. Betti, B. Hu, R. E. Bell, S. P. Gerhardt, J. Manickam, and K. Tritz, Phys. Rev. Lett. 104, 035003 (2010).

${ }^{7}$ B. Coppi, Nucl. Fusion 42, 1 (2002).

${ }^{8}$ A. G. Peeters and C. Angioni, Phys. Plasmas 12, 072515 (2005).

${ }^{9}$ A. G. Peeters, C. Angioni, and D. Strintzi, Phys. Rev. Lett. 98, 265003 (2007).

${ }^{10}$ T. S. Hahm, P. H. Diamond, O. D. Gurcan, and G. Rewoldt, Phys. Plasmas 14, 072302 (2007).

${ }^{11}$ D. Strintzi, A. G. Peeters, and J. Weiland, Phys. Plasmas 15, 044502 (2008).

${ }^{12}$ J. Weiland, R. Singh, H. Nordman, P. Kaw, A. G. Peeters, and D. Strinzi, Nucl. Fusion 49, 065033 (2009).

${ }^{13}$ D. Nishijima, A. Kallenbach, S. Günter, M. Kaufmann, K. Lackner, C. F. Maggi, A. G. Peeters, G. V. Pereverzev, B. Zaniol, and ASDEX Upgrade Team, Plasma Phys. Controlled Fusion 47, 89 (2005).

${ }^{14}$ J. S. de Grassie, D. R. Baker, K. H. Burrell, P. Gohil, C. M. Greenfield, R. J. Groebner, and D. M. Thomas, Nucl. Fusion 43, 142 (2003).

${ }^{15}$ P. C. de Vries, K. M. Rantamäki, C. Giroud, E. Asp, G. Corrigan, A. Eriksson, M. de Greef, I. Jenkins, H. C. M. Knoops, P. Mantica, H. Nordman, P. Strand, T. Tala, J. Weiland, K.-D. Zastrow, and JET EFDA Contributors, Plasma Phys. Controlled Fusion 48, 1693 (2006).

${ }^{16}$ P. C. de Vries, M.-D. Hua, D. C. McDonald, C. Giroud, M. Janvier, M. F. Johnson, T. Tala, K.-D. Zastrow, and JET EFDA Contributors, Nucl. Fusion 48, 065006 (2008).

${ }^{17}$ J. E. Rice, A. Ince-Cushman, J. S. deGrassie, L.-G. Eriksson, Y. Sakamoto, A. Scarabosio, A. Bortolon, K. H. Burrell, B. P. Duval, C. Fenzi-Bonizec, M. J. Greenwald, R. J. Groebner, G. T. Hoang, Y. Koide, E. S. Marmar, A. Pochelon, and Y. Podpaly, Nucl. Fusion 47, 1618 (2007).

${ }^{18}$ L.-G. Eriksson, T. Hellsten, M. F. F. Nave, J. Brzozowski, K. Holmström, T. Johnson, J. Ongena, K.-D. Zastrow, and JET-EFDA Contributors, Plasma Phys. Controlled Fusion 51, 044008 (2009).

${ }^{19}$ A. Jacchia, F. De Luca, G. Gorini, and P. Mantica, Phys. Fluids B 3, 3033 (1991).

${ }^{20}$ P. Mantica and F. Ryter, C. R. Phys. 7, 634 (2006).

${ }^{21}$ K. Nagashima, Y. Koide, and H. Shirai, Nucl. Fusion 34, 449 (1994).

${ }^{22}$ M. Yoshida, Y. Koide, H. Takenaga, H. Urano, N. Oyama, K. Kamiya, Y.
Sakamoto, G. Matsunaga, Y. Kamada, and JT-60 Team, Nucl. Fusion 47, 856 (2007).

${ }^{23}$ T. Tala, K.-D. Zastrow, J. Ferreira, P. Mantica, V. Naulin, A. G. Peeters, G. Tardini, M. Brix, G. Corrigan, C. Giroud, and D. Strintzi, Phys. Rev. Lett. 102, 075001 (2009).

${ }^{24}$ G. Tardini, J. Ferreira, P. Mantica, A. G. Peeters, T. Tala, K. D. Zastrow, M. Brix, C. Giroud, G. V. Pereverzev, and JET-EFDA Contributors, Nucl. Fusion 49, 085010 (2009).

${ }^{25}$ T. Tala, K. Crombé, P. C. de Vries, J. Ferreira, P. Mantica, A. G. Peeters, Y. Andrew, R. Budny, G. Corrigan, A. Eriksson, X. Garbet, C. Giroud, M.-D. Hua, H. Nordman, V. Naulin, M. F. F. Nave, V. Parail, K. Rantamäki, B. D. Scott, P. Strand, G. Tardini, A. Thyagaraja, J. Weiland, K.-D. Zastrow, and JET-EFDA Contributors, Plasma Phys. Controlled Fusion 49, B291 (2007).

${ }^{26}$ W. M. Solomon, K. H. Burrell, A. M. Garofalo, S. M. Kaye, R. E. Bell, A. J. Cole, J. S. deGrassie, P. H. Diamond, T. S. Hahm, G. L. Jackson, M. J. Lanctot, C. C. Petty, H. Reimerdes, S. A. Sabbagh, E. J. Strait, T. Tala, and R. E. Waltz, Phys. Plasmas 17, 056108 (2010).

${ }^{27}$ W. M. Solomon, K. H. Burrell, A. M. Garofalo, A. J. Cole, R. V. Budny, J. S. deGrassie, W. W. Heidbrink, G. L. Jackson, M. J. Lanctot, R. Nazikian, H. Reimerdes, E. J. Strait, M. A. Van Zeeland, and DIII-D Rotation Physics Task Force, Nucl. Fusion 49, 085005 (2009).

${ }^{28}$ W. M. Solomon, S. M. Kaye, R. E. Bell, B. P. LeBlanc, J. E. Menard, G. Rewoldt, W. Wang, F. M. Levinton, H. Yuh, and S. A. Sabbagh, Phys. Rev. Lett. 101, 065004 (2008).

${ }^{29}$ S. M. Kaye, W. Solomon, R. E. Bell, B. P. LeBlanc, F. Levinton, J. Menard, G. Rewoldt, S. Sabbagh, W. Wang, and H. Yuh, Nucl. Fusion 49, 045010 (2009).

${ }^{30}$ K.-D. Zastrow, W. G. F. Core, L.-G. Eriksson, M. G. Von Hellermann, A. C. Howman, and R. W. T. König, Nucl. Fusion 38, 257 (1998).

${ }^{31}$ C. R. Negus, C. Giroud, A. G. Meigs, K.-D. Zastrow, and D. L. Hillis, Rev. Sci. Instrum. 77, 10F102 (2006).

${ }^{32}$ R. J. Goldston, D. C. McCune, H. H. Towner, S. L. Davis, R. J. Hawryluk, and G. L. Schmidt, J. Comput. Phys. 43, 61 (1981).

${ }^{33}$ L. L. Lao, H. E. St. John, R. D. Stambaugh, A. G. Kellman, and W. Pfeiffer, Nucl. Fusion 25, 1611 (1985).

${ }^{34}$ A. Pankin, D. McCune, R. Andre, G. Bateman, and A. Kritz, Comput. Phys. Commun. 159, 157 (2004).

${ }^{35}$ J. A. Heikkinen and S. K. Sipilä, Phys. Plasmas 2, 3724 (1995).

${ }^{36}$ M. Erba, A. Cherubini, V. V. Parail, E. Springmann, and A. Taroni, Plasma Phys. Controlled Fusion 39, 261 (1997).

${ }^{37}$ P. Mantica, D. Strintzi, T. Tala, C. Giroud, T. Johnson, H. Leggate, E. Lerche, T. Loarer, A. G. Peeters, A. Salmi, S. Sharapov, D. Van Eester, P. C. de Vries, L. Zabeo, and K.-D. Zastrow, Phys. Rev. Lett. 102, 175002 (2009).

${ }^{38}$ F. Ryter, C. Angioni, C. Giroud, A. G. Peeters, T. Biewer, R. Bilato, J. Candy, E. Joffrin, T. Johnson, H. Leggate, E. Lerche, G. Madison, P. Mantica, D. Van Eester, I. Voitsekhovitch, R. E. Waltz, and JET Contributors, Proceedings of the 22nd IAEA Fusion Energy Conference, Geneva, 2008 (IAEA, Vienna, 2008).

${ }^{39}$ X. Garbet, P. Mantica, F. Ryter, G. Cordey, F. Imbeaux, C. Sozzi, A. Manini, E. Asp, V. Parail, R. Wolf, and JET EFDA Contributors, Plasma Phys. Controlled Fusion 46, 1351 (2004).

${ }^{40} \mathrm{~J}$. Weiland, Collective Modes in Inhomogeneous Plasmas (IOP, Bristol, 2000).

${ }^{41}$ A. G. Peeters, C. Angioni, A. Bottino, A. Kallenbach, B. Kurzan, C. F. Maggi, W. Suttrop, and ASDEX Upgrade Team, Plasma Phys. Controlled Fusion 48, B413 (2006).

${ }^{42}$ A. G. Peeters, D. Strintzi, Y. Camenen, C. Angioni, F. J. Casson, W. A. Hornsby, and A. P. Snodin, Phys. Plasmas 16, 042310 (2009).

${ }^{43}$ M. B. Isichenko, A. V. Gruzinov, and P. H. Diamond, Phys. Rev. Lett. 74, 4436 (1995).

${ }^{44}$ A. G. Peeters, C. Angioni, and D. Strintzi, Phys. Plasmas 16, 034703 (2009).

${ }^{45}$ A. G. Peeters, Y. Camenen, F. J. Casson, W. A. Hornsby, A. P. Snodin, D. Strintzi, and G. Szepesi, Comput. Phys. Commun. 180, 2650 (2009).

${ }^{46}$ A. G. Peeters, C. Angioni, Y. Camenen, F. J. Casson, W. A. Hornsby, A. P. Snodin, and D. Strintzi, Phys. Plasmas 16, 062311 (2009).

${ }^{47}$ R. R. Dominguez and G. M. Staebler, Phys. Fluids B 5, 3876 (1993).

${ }^{48}$ Y. Camenen, A. G. Peeters, C. Angioni, F. J. Casson, W. A. Hornsby, A. P. Snodin, and D. Strintzi, Phys. Rev. Lett. 102, 125001 (2009).

${ }^{49}$ P. H. Diamond, C. J. McDevitt, Ö. D. Gürcan, T. S. Hahm, and V. Naulin, Phys. Plasmas 15, 012303 (2008).

${ }^{50}$ Fusion Sci. Technol. 53(4) (2008). 